\title{
How Many Consumers are Rational?
}

\author{
Stefan Hoderlein* \\ Brown University
}

October 20, 2009

\begin{abstract}
Rationality places strong restrictions on individual consumer behavior. This paper is concerned with assessing the validity of the integrability constraints imposed by standard utility maximization, arising in classical consumer demand analysis. More specifically, we characterize the testable implications of negative semidefiniteness and symmetry of the Slutsky matrix across a heterogeneous population without assuming anything on the functional form of individual preferences. In the same spirit, homogeneity of degree zero is being considered. Our approach employs nonseparable models and is centered around a conditional independence assumption, which is sufficiently general to allow for endogenous regressors. It is the only substantial assumption a researcher has to specify in this model, and has to be evaluated with particular care. Finally, we apply all concepts to British household data: We show that rationality is an acceptable description for large parts of the population, regardless of whether we test on single or composite households.
\end{abstract}

*Department of Economics, Brown University, Box B, Providence, RI 02912. email: stefan_hoderlein "at" yahoo.com. 
JEL Codes: C12, C14, D12, D01.

Keywords: Nonparametric, Integrability, Testing Rationality, Nonseparable Models, Demand, Nonparametric IV.

\section{Introduction}

Economic theory yields strong implications for the actual behavior of individuals. In the standard utility maximization model for instance, economic theory places important restrictions on individual responses to changes in prices and wealth, the so-called integrability constraints. However, when we want to evaluate the validity of the integrability conditions using real data, we face the following problem: In a typical consumer data set we observe individuals only under a very limited number of different price-wealth combinations, often only once. Consequently, the observations of "comparable" individuals have to be taken into consideration. But this conflicts with the important notion of unobserved heterogeneity, a notion that is supported by the widespread empirical finding that individuals with the same covariates vary dramatically in their actual consumption behavior. Thus, general and unrestrictive models for handling unobserved heterogeneity are essential for testing integrability.

Endogeneity is another major source of concern in applied work. In consumer demand, total expenditure is taken as income concept, which is justified by assuming intertemporal separability of preferences. Since the categories of goods considered are broad (e.g., food) and they frequently constitute a large part of total expenditure, the latter is believed to be endogenous. As instrument, the demand literature usually employs labor income whose determinants are thought to be exogenous to the unobserved preferences determining, say, food consumption. Other endogeneities that could arise are in particular related to prices. In empirical industrial 
organization for instance, prices for individual goods are thought to be set by the firm targeting (partially unobserved) characteristics of individual consumers. The arising endogeneities could be tackled in exactly the same fashion as we propose in this paper. In our application, however, we consider broad aggregates of goods and we expect such effects to wash out. Moreover, following the recent Microeconometric demand literature we consider atomic individuals that act as price takers, and we control for time effects. Summarizing, we concentrate on total expenditure endogeneity, but add that this approach could easily be extended.

Testing integrability constraints dates back at least to the early work of Stone (1954), and has spurned the extensive research on (parametric) flexible functional forms demand systems (e.g., the Translog, Jorgenson et al. (1982), and the Almost Ideal, Deaton and Muellbauer (1980)). Nonparametric analysis of some derivative constraints was performed by Stoker (1989) and Härdle, Hildenbrand and Jerison (1991), but none of these has its focus on modeling unobserved heterogeneity. More closely related to our approach is Lewbel (2001) who analyzes integrability constraints in a purely exogenous setting. In comparison to his work, we make three contributions: First, we show how to handle endogeneity. Second, even restricted to the exogenous case, some of our results (e.g., on negative semidefiniteness) are new and more general. Third, we propose, discuss and implement nonparametric test statistics. An alternative method for checking some integrability constraints is revealed preference analysis, see Blundell, Browning and Crawford (2003), and references therein.

In this paper we extend the recent work on nonseparable models - in particular Imbens and Newey (2003) and Altonji and Matzkin (2005) who treat the estimation of average structural derivatives when regressors are endogenous - to testing integrability conditions. Even though our application comes from traditional consumer demand, most of the analysis is by no means confined to this setup and could be applied to any standard utility maximization problem with 
linear budget constraints. Moreover, the spirit of the analysis could be extended to cover, e.g., decisions under uncertainty or nonlinear budget constraints.

Central to this paper is a conditional independence assumption. This assumption will be the only material restriction we place on preference heterogeneity, and it explicitly allows for endogenous regressors. Since this is the major structural assumption, it has to scrutinized in any specific application. In our application, price variation is only available over time, and not, as would be preferable, in the cross section dimension. This has the consequence that we have to pool several cross sections, in which case the structural independence assumption effectively requires time invariance of the distribution of preferences. To mitigate this effect (which equally plagues parametric models that use the same data), we allow for a time trend in a nonparametric fashion. However, given the typical bandwidth chosen in our application this corresponds implicitly to assuming stability of the preference ordering over a period of approximately five years. While we may do better with improved data (e.g., data that contains also cross section price information), the upshot is that this conditional independence assumption is not as unrestrictive as it may seem. Specifically, its restrictiveness has to be assessed in the light of any given set of data, and the data set we (and much of the applied literature) uses is clearly restrictive in terms of price variation.

Based on this independence assumption, the main contribution of this paper is the formal clarification of the implications of this conditional independence assumption for testing integrability constraints with data. Much of the second section will be specifically devoted to this issue: we devise very general tests for Slutsky negative semidefiniteness and symmetry, as well as for homogeneity. In the third section we apply these concepts to british FES data. The results are affirmative as far as the validity of the integrability conditions are concerned. One feature of our approach is that we are able to look in detail at various subpopulations 
of interest, and hence shed light on potential sources of rejections of rationality. To give a particular example, we consider a question that has recently achieved a great deal of attention in the demand literature, namely whether estimating demand models using household data is not fundamentally flawed because it neglects household composition effects see, e.g., Browning and Chiappori (1998), Fortin and Lacroix (1997) and Cherchye and Vermeulen (2008). To analyze the hypothesis that rejections of rationality using household data are attributable to, say, bargaining behavior amongst individually rational household members, we look at single households and two person households separately. The results are somewhat surprising in the sense that we do not find two person households to be less rational than singles.

After this empirical exercise, we conclude this paper with a summary and an outlook. The appendix contains proofs, graphs and summary statistics. A more detailed description of the econometric methods may be obtained from a supplement that is available on the author's webpage.

\section{The Demand Behavior of a Heterogeneous Population}

\subsection{Structure of the Model and Assumptions}

Our model of consumer demand in a heterogeneous population consists of several building blocks. As is common in consumer demand, we assume that - for a fixed preference ordering there is a causal relationship between budget shares, a $[0,1]$ valued random $L$-vector denoted by $W$, and regressors of economic importance, namely log prices $P$ and log total expenditure $Y$, real valued random vectors of length $L$ and 1 , respectively. Let $X=\left(P^{\prime}, Y\right)^{\prime} \in \mathbb{R}^{L+1}$. To capture the notion that preferences vary across the population, we assume that there is 
a random variable $V \in \mathcal{V}$, where $\mathcal{V}$ is a Borel space ${ }^{1}$, which denotes preferences (or more generally, decision rules). We assume that heterogeneity in preferences is partially explained by observable differences in individuals' attributes (e.g., age), which we denote by the real valued random $G$-vector $Q$. Hence, we let $V=\vartheta(Q, A)$, where $\vartheta$ is a fixed $\mathcal{V}$-valued mapping defined on the sets $\mathcal{Q} \times \mathcal{A}$ of possible values of $(Q, A)$, and where the random variable $A$ (taking again values in a Borel space $\mathcal{A}$ ) covers residual unobserved heterogeneity in a general fashion.

What can we learn from cross section data about individual rationality in a heterogeneous population? To understand the limits of identification in this setup, we start by considering a heterogeneous linear population, i.e., neglecting the dependence on $Q$, we assume for the moment that the outcome equation were given by a random coefficients model,

$$
W=X^{\prime} A_{1}
$$

where $A_{1}$ denotes the preference parameters that vary across the population ${ }^{2}$. In Hoderlein, Klemelä and Mammen (2009), we show that the distribution $f_{A_{1}}$ is nonparametrically identified by inverting an integral equation, but even in this case we require strong and economically not very plausible necessary conditions for identification like fat tailed distributions of regressors, and obtain a slow rate of convergence due to the regularization involved in solving the integral equation. Hence, we could argue that $f_{A_{1}}$ is only poorly identified in the linear heterogeneous population, and an "one-type", parametric, but nonlinear, population is only weakly identified in general as the rates of convergence may be too slow to allow estimation with any reasonable

\footnotetext{
${ }^{1}$ Technically: $\mathcal{V}$ is a set that is homeomorphic to the Borel subset of the unit interval endowed with the Borel $\sigma$-algebra. This includes the case when $V$ is an element of a polish space, e.g., the space of random piecewise continuous utility functions.

${ }^{2}$ In consumer demand, this corresponds approximately to an Almost Ideal demand system, with the standard shortcut of setting the denominator terms in the income effect equal to a price index.
} 
amount of data. Moreover, this model is quite restrictive in terms of the heterogeneity it allows, and we would like to be able to be much more general: Ideally, we would allow not just for one but for (infinitely) many types, and for the parameters to be not necessarily finite but infinite dimensional. Therefore we formalize the heterogeneous population as $W=\phi(X, A)$, for a general mapping $\phi$ and an infinite dimensional vector $A$. Obviously, neither $\phi$ nor $f_{A}$ are nonparametrically identified. Still, for any fixed value of $A$, say $a_{0}$, we obtain a demand function having standard properties, and the hope is that when forming some type of average over the unobserved heterogeneity $A$ (or using some other type of operation), rationality properties of individual demand may be preserved by some structure.

As mentioned, a contribution of this paper is that we allow for dependence between unobserved heterogeneity $A$ and all regressors of economic interest. To this end, we introduce the real valued random $K$-vector of instruments $S$. Note that $S$ may contain exogenous elements in $X$, which serve as their own instruments.

Having defined all elements of our model, we are now in the position to state it formally, including observable covariates $Q$ :

Assumption 2.1 Let all variables be defined as above. The formal model of consumer demand in a heterogeneous population is given by

$$
\begin{aligned}
W & =\phi(X, \vartheta(Q, A)) \\
X & =\mu(S, Q, U)
\end{aligned}
$$

where $\phi$ is a fixed $\mathbb{R}^{L}$-valued Borel mapping defined on the sets $\mathrm{X} \times \mathcal{V}$ of possible values of $(X, V)$. Analogously, $\mu$ is a fixed $\mathbb{R}^{L+1}$-valued Borel mapping defined on the sets $\mathcal{S} \times \mathcal{Q} \times \mathcal{U}$ of possible values of $(S, Q, U)$. Moreover, $\mu$ is assumed to be invertible in $U$, for every value of $(S, Q)$. 
Assumption 2.1 defines the nonparametric demand system with (potentially) endogenous regressors as a system of nonseparable equations. these models are called nonseparable, because they do not impose an additive specification for the unobservable random terms (in our case $A$ or $U$ ). Note that in the case of endogeneity of $X$ this requires that $U$ be solved for, because the residuals have to be employed actively in a control function fashion. In the application, we specify $\mu$ to be the conditional mean function, and consequently $U$ to be additive mean regression residuals. However, identification proceeds more generally: In the single endogenous regressor case, $\mu$ could be the median regression and $U$ the median regression residuals. In the case of several endogeneous regressors, $\mu$ could be a triangular system of nonseparable functions, or a location-scale model. To allow the applied researcher to select the most appropriate specification, we therefore do not restrict the specification at this point.

Nonseparable models have been subject of much interest in the recent econometrics literature (Chesher (2003), Matzkin (2003), Altonji and Matzkin (2005), Imbens and Newey (2003), Hoderlein and Mammen (2007), to mention just a few). Since we do not assume monotonicity in unobservables, our approach is more closely related to the latter three approaches. As is demonstrated there, in the absence of strict monotonicity of $\phi$ in $A$, the function $\phi$ is not identified, however, local average structural derivatives are. These models are ideal in our application, as they allow assessing integrability constraints without taking into account the limitations associated with some of the parametric specifications ${ }^{3}$.

Writing $Z=\left(Q^{\prime}, U\right)^{\prime}$, we formalize the notion of independence between instruments and unobservables as follows:

\footnotetext{
${ }^{3}$ For instance, the frequently employed approximate AI demand system is not integrable. I am indebted to a referee for pointing this out.
} 
Assumption 2.2 Let all variables be as defined above. Then we require that

$$
F_{A \mid S, Z}=F_{A \mid Z}
$$

There are also some differentiability and regularity conditions involved, which are summarized in the appendix in assumption 2.3. However, assumption 2.2 is the key identification assumption and merits a thorough discussion: Assume for a moment all regressors were exogenous, i.e. $S \equiv X$ and $U \equiv 0$. Then this assumption states that $X$, in our case wealth and prices, and unobserved heterogeneity are independently distributed, conditional on individual attributes.

To give an example: Suppose that in order to determine the effect of wealth on consumption, we are given data on the demand of individuals, their wealth and the following attributes: "education in years" and "gender". Take now a typical subgroup of the population, e.g., females having received 12 years of education. Assume that there be two wealth classes for this subgroup, rich and poor, and two types of preferences, type 1 and 2 . Then, for both rich and poor women in this subgroup, the proportion of type 1 and 2 preferences has to be identical. This assumption is of course restrictive. Note, however, that $A$ (the infinite dimensional preference parameter) and regressors of economic interest may still be correlated unconditionally across the population. Moreover, any of the $Z$ may be arbitrarily correlated with $A$.

Now turn to the case of endogenous regressors and instruments. Suppose we were again interested in the effect of changes in wealth on the demand of an individual. In the demand literature, wealth equals total expenditure, but the latter is commonly assumed to be endogenous, and hence labor income, denoted by $S_{1}$, is taken as an instrument. In a world of rational agents, labor income is the result of maximizing behavior by individual agents (consumers and firms). Much as above, we can model $S_{1}=v\left(Q, A_{2}\right)$, where $v$ is a fixed Borel-measurable scalar valued 
function defined on the set $\mathcal{Q} \times \mathcal{A}_{2}$. Here, $A_{2}$ is a set of unobservables containing variables that govern the decision of the individual's intertemporal optimal labor supply problem, e.g. the attitude towards risk or idiosyncratic information sets. Note that $Q$ may contain variables that determine both the labor supply, as well as the demand decision like household characteristics. But the wage rate could also be contained in $Q$, without however having an influence on $\phi$ (i.e the derivative of $\vartheta$ with respect to the wage rate is zero because of intertemporal separability).

First, consider the extreme case where $A$ and $A_{2}$ are independent conditional on $Q$. In addition to independence between $U$ and $S_{1}$, we assume that $U$ is not a function of $S_{1}$, i.e. $U=\varphi(Q, A)$, , where $v$ is a fixed Borel-measurable scalar valued function defined on the set $\mathcal{Q} \times \mathcal{A}$. Then, assumption 2.3 is quite plausible as in this case the condition $F_{A \mid S_{1}, Z}=F_{A \mid Z}$ i.e., $F_{A \mid v\left(Q, A_{2}\right), \varphi(Q, A), Q}=F_{A \mid \varphi(Q, A), Q}$, can be derived as follows:

$$
F_{A \mid v\left(Q, A_{2}\right), \varphi(Q, A), Q}=\frac{F_{A, v\left(Q, A_{2}\right), \varphi(Q, A) \mid Q}}{F_{v\left(Q, A_{2}\right), \varphi(Q, A) \mid Q}}=\frac{F_{A, \varphi(Q, A) \mid v\left(Q, A_{2}\right), Q}}{F_{\varphi(Q, A) \mid Q}}=\frac{F_{A, \varphi(Q, A) \mid Q}}{F_{\varphi(Q, A) \mid Q}}=F_{A \mid \varphi(Q, A), Q}
$$

where only the conditional independence between $A$ and $A_{2}$ has been used.

In contrast, assume that $A=A_{2}$, i.e. the unobservable characteristics of the household that govern the two decisions are the same. Then both $S_{1}$ and $U$ are functions of $A$. Nevertheless, $F_{A \mid S_{1}, Z}=F_{A \mid Z}$ is still not completely implausible as $U$ already reflects some influence of $A$.

As mentioned in the introduction, this assumption is potentially restrictive in any specific application due to limitations of the data. In our application for instance, we pool observations from twenty time years ${ }^{4}$. To avoid assuming stability of preferences over 20 years, we include a time trend in the set of conditioning variables. Since we employ kernel based nonparametric regression techniques, our choice of smoothing parameters result in effectively pooling observations from five adjacent years on average; it is over such a time horizon that we have to assume

\footnotetext{
${ }^{4}$ Particularly, we use monthly data on aggregate prices, which exhibit largely time series variation.
} 
stability of the distribution of preferences over time. This gives an idea of the sense in which the content of this assumption has to be checked in any specific application. Limitations to the quality of the data show up at this point in our framework, and we encounter the familiar finding from parametric models that the less good the quality of the data, the less confident we can be in our main identifying assumptions.

\subsection{Implications for Observable Behavior}

Given these assumptions and notations, we concentrate first on the relation of theoretical quantities and the identified objects, specifically $m(\xi, z)=\mathbb{E}[W \mid X=\xi, Z=z]$ and $M(s, z)=$ $\mathbb{E}[W \mid S=s, Z=z]$ which denote the conditional mean regression function using either endogenous regressors and controls, or instruments and controls. Finally, let $\sigma\{X\}$ denote the information set ( $\sigma$-algebra) spanned by $X$, and $\Xi^{-}$be the Moore-Penrose pseudo inverse of a matrix $\Xi$.

More specifically, we focus on the following questions:

1. How are the identified marginal effects (i.e., $D_{x} m$ or $D_{x} M$ ) related to the theoretical derivatives $D_{x} \phi ?$

2. How and under what kind of assumptions do observable elements allow inference on key elements of economic theory? Especially, what do we learn about homogeneity, adding up, as well as negative semidefiniteness and symmetry of the Slutsky matrix, which in the standard consumer demand setup we consider (with budget shares as dependent variables, and log prices and log total expenditure as regressors), and in the underling heterogeneous population (defined by $\phi, x$, and $v$ ), takes the form

$$
\mathfrak{S}(x, v)=D_{p} \phi(x, v)+\partial_{y} \phi(x, v) \phi(x, v)^{\prime}+\phi(x, v) \phi(x, v)^{\prime}-\operatorname{diag}\{\phi(x, v)\} .
$$


Here, $\operatorname{diag}\{\phi\}$ denotes the matrix having the $\phi_{j}, j=1, . ., L$ on the diagonal and zero off the diagonal. The second question shall be the subject of Propositions 2.2 and 2.3. We start, however, with Lemma 2.1 which answers the question on the relationship between estimable and theoretical derivatives. All proofs may be found in the appendix.

Lemma 2.1 Let all the variables and functions be as defined above. Assume that A2.1-A2.3 hold. Then,

$$
\begin{aligned}
& \text { (i) } \mathbb{E}\left[D_{x} \phi(X, V) \mid X, Z\right]=D_{x} m(X, Z) \quad \text { (a.s.), } \\
& \text { (ii) } \mathbb{E}\left[D_{x} \phi(X, V) \mid S, Z\right]=D_{s} M(S, Z) D_{s} \mu(S, Z)^{-} \quad \text { (a.s.). }
\end{aligned}
$$

$A d(i)$ : This result establishes that every individual's empirically obtained marginal effect is the best approximation (in the sense of minimizing distance with respect to $L_{2}$-norm) to the individual's theoretical marginal effect. Note that it is only the local conditional average of the derivative of $\phi$ that may be identified and not the function $\phi$. Suppose we were given data on consumption, total expenditure, "education in years" and "gender" as above. Then by use of the marginal effect $D_{x} m(X, Z)$, we may identify the average marginal total expenditure effect on consumption of, e.g., all female high school graduates, but not the marginal effect of every single individual. Arguably, for most purposes the average effect on the female graduates is all that decision makers care about.

Ad (ii) : This result illustrates that assumption $A 2.2$ has several implications on observables depending which conditioning set is used. As the preceding discussion shows, conditioning on $X, Z$ seems natural as the subgroups formed have a direct economic interpretation. However, recall that $\sigma\{X, Z\} \subseteq \sigma\{S, Z\}$. Hence, $\sigma\{S, Z\}$ should be employed, because conditional expectations using $\sigma\{S, Z\}$ are closer in $L_{2}$-norm to the true derivatives. Altonji and Matzkin (2005) derive an estimator for $\mathbb{E}\left[D_{x} \phi(X, V) \mid X, Z\right]$ by integrating $(i)$ over $U$, but conditioning on $X, Z$. Since our focus is on testing economic restrictions, we avoid the integration step as 
in many cases it reduces the power of all test statistics. Therefore we give always results using $\sigma\{X, Z\}$ and $\sigma\{S, Z\}$. The former has a more clear cut economic interpretation, the latter yields tests of higher power.

We now turn to the question which economic properties in a heterogeneous population have testable counterparts. This problem bears some similarities with the literature on aggregation over agents in demand theory, because taking conditional expectations can be seen as an aggregation step. We introduce the following notation: Let $e_{j}$ denote the $j$-th unit vector of length $L+1$, let $E_{j}=\left(e_{1}, . ., e_{j}, 0, . ., 0\right)$ and $\iota$ be the vector containing only 1.

Now we are in the position to state the following proposition, which is concerned with adding up and homogeneity of degree zero, two properties which are related to the linear budget set:

Proposition 2.2 Let all the variables and functions be defined as above, and suppose that A2.1 and A2.3 hold. Then, $\iota^{\prime} \phi=1$ (a.s.) $\Rightarrow \iota^{\prime} m=1$ and $\iota^{\prime} M=1$ (a.s.). If, in addition, $F_{A \mid X, Z}(a, \xi, z)=F_{A \mid X, Z}(a, \xi+\lambda, z)$ is true for all $\xi \in X$ and $\lambda>0$, then

$$
\phi(X, V)=\phi(X+\lambda, V) \text { (a.s.) } \Rightarrow m(X, Z)=m(X+\lambda, Z) \quad \text { (a.s.). }
$$

$\operatorname{Under}(A 2.1)-(A 2.3)$ we obtain that,

$$
\begin{aligned}
\phi(X, V) & =\phi(X+\lambda, V)(\text { a.s. }) \Rightarrow D_{p} m \iota+\partial_{y} m=0 \text { (a.s.) } \\
\text { and } \phi(X, V) & =\phi(X+\lambda, V) \text { (a.s.) } \Rightarrow D_{s} M D_{s} \mu^{-} E_{L} \iota+D_{s} M D_{s} \mu^{-} e_{L+1}=0 \text { (a.s.). }
\end{aligned}
$$

Finally, if we also assume $(A 2.1),(A 2.3), \mu(S, Z)=\mu(S+\lambda, Z)$, as well as,

$$
\begin{aligned}
& F_{A \mid S, Z}(a, s, z)=F_{A \mid S, Z}(a, s+\lambda, z) \text {, then } \\
& \qquad(X, V)=\phi(X+\lambda, V) \quad(\text { a.s. }) \Rightarrow M(S, Z)=M(S+\lambda, Z) \text { (a.s.) }
\end{aligned}
$$

Remark 2.2: Note that we do not require any type of independence for adding up to carry through to the world of observables. This is very comforting since - in the absence of any 
direct way of testing this restriction - adding up is imposed on the regressions by deleting one equation.

The homogeneity part of Proposition 2.2 is ordered according to the severity of assumptions: Homogeneity carries through to the regression conditioning on endogenous regressors and controls under a homogeneity assumption on the cdf. This assumption is weaker than $A 2.2$ as it is obviously implied by conditional independence. The derivative implications are generally true under conditional independence. This is particularly useful for testing homogeneity using the regression including instruments, as for this regression to inherit homogeneity an implausible additional homogeneity assumption, $\mu(S, Z)=\mu(S+\lambda, Z)$, has to be fulfilled.

The following proposition is concerned with the Slutsky matrix. We need again some notation. Let $\mathbb{V}[G, H \mid \mathcal{F}]$ denote the conditional covariance matrix between two random vectors $G$ and $H$, conditional on some $\sigma$-algebra $\mathcal{F}$, and $\mathbb{V}[H \mid \mathcal{F}]$ be the conditional covariance matrix of a random vector $H$. We will also make use of the second moment regressions, i.e. $m_{2}(\xi, z)=\mathbb{E}\left[W W^{\prime} \mid X=\xi, Z=z\right]$ and $M_{2}(s, z)=\mathbb{E}\left[W W^{\prime} \mid S=s, Z=z\right]$. Moreover, denote by vec the operator that stacks a $m \times q$ matrix columnwise into a $m q \times 1$ column vector, and by $v e c^{-1}$ the operation that stacks an $m q \times 1$ column vector columnwise into a $m \times q$ matrix. We also abbreviate negative semidefiniteness by $n s d$. Finally, for any square matrix $B$, let $\bar{B}=B+B^{\prime}$

Proposition 2.3: Let all the variables and functions be defined as above. Suppose that A2.1 -

A2.3 hold. Then, the following implications hold almost surely:

$\mathfrak{S} n s d \Rightarrow \overline{D_{p} m}+\partial_{y} m_{2}+2\left(m_{2}-\operatorname{diag}\{m\}\right) n s d$, and

$\mathfrak{S} n s d \Rightarrow \overline{D_{s} M D_{s} \mu^{-} E_{L}}+\operatorname{vec}^{-1}\left\{D_{s} v e c\left[M_{2}\right] D_{s} \mu^{-} e_{L+1}\right\}+2\left(M_{2}-\operatorname{diag}\{M\}\right) n s d$ However, if and only if $\mathbb{V}\left[\partial_{y} \phi, \phi \mid X, Z\right]$, respectively $\mathbb{V}\left[\partial_{y} \phi, \phi \mid S, Z\right]$, are symmetric we have 
$\mathfrak{S}$ symmetric $\Rightarrow D_{p} m+\partial_{y} m m^{\prime}$ symmetric, and

$\mathfrak{S}$ symmetric $\Rightarrow D_{s} M D_{s} \mu^{-} E_{L}+D_{s} M D_{s} \mu^{-} e_{L+1} M^{\prime} \quad$ symmetric

almost surely.

Remark 2.3: The importance of this proposition lies in the fact that it allows for testing the key elements of rationality without having to specify the functional form of the individual demand functions or their distribution in a heterogeneous population. Indeed, the only element that has to be specified correctly is a conditional independence assumption.

Suppose now we see any of these conditions rejected in the observable (generally nonparametric) regression at a position $x, z$. Recalling the interpretation of the conditional expectation as average (over a "neighborhood") this proposition tells us that there exists a set of positive measure of the population ("some individuals in this neighborhood") which does not conform with the postulates of rationality. An interesting question is when the reverse implications hold as well, i.e. one can deduce from the properties of the observable elements directly on $\mathfrak{S}$. This issue is related to the concept of completeness raised in Blundell, Chen and Kristensen (2007). It is our conjecture that some of the concepts may be transferred, but a detailed treatment is beyond the scope of this paper and will be left for future research. The basic intuition for the results are that expectations are linear operators in the functions involved, implying that many properties on individual level remain preserved. However, difficulties arise when nonlinearities are present, as is the case with conventional tests for Slutsky symmetry. The fact that we are able to circumvent this problem in the case of negative semidefiniteness relies on the fact that we may able to transform the property on individual level so that it is linear in the squared regression.

Proposition 2.3 illustrates clearly that appending "an additive error capturing unobserved 
heterogeneity" and proceeding as if the mean regression $m$ has the properties of individual demand is not the way to solve the problem of unobserved heterogeneity. Note that we may always append a mean independent additive error, since $\phi=m+(\phi-m)=m+\varepsilon$. The crux is now that the error is generally a function of $y$ and $p$. For instance, the potentially nonsymmetric part of the Slutsky matrix becomes

$$
\mathfrak{S}=D_{p} m+\partial_{y} m m^{\prime}+D_{p} \varepsilon+\left(\partial_{y} m\right) \varepsilon^{\prime}+\left(\partial_{y} \varepsilon\right) m^{\prime}+\left(\partial_{y} \varepsilon\right) \varepsilon^{\prime},
$$

and the last four terms will not vanish in general.

Returning to Proposition 2.3, one should note a key difference between negative semidefiniteness and symmetry. For the former we may provide an "if" characterization without any assumptions other than the basic ones. To obtain a similar result for symmetry, we have to invoke an additional assumption about the conditional covariance matrix $\mathbb{V}\left[\partial_{y} \phi, \phi \mid X, Z\right]$. This matrix is unobservable - at least without any further identifying assumptions. Note that this assumption is (implicitly) implied in all of the demand literature, since symmetry is inherited by $D_{p} m+\partial_{y} m m^{\prime}$ only under this assumption.

Conversely, if this additional assumption does not hold, we are able to test at most for homogeneity, adding up and Slutsky negative semidefiniteness. This amounts to demand behavior generated by complete, but not necessarily transitive preferences. Details of this demand theory of the weak axiom can be found in Kihlstrom, Mas-Colell and Sonnenschein (1976) and Quah (2000). Furthermore, note some parallels with the aggregation literature in economic theory: Only adding up and homogeneity carry immediately through to the mean regression. This result is similar in spirit to the Mantel-Sonnenschein theorem, where only these two properties are inherited by aggregate demand. It is also well known in this literature that the aggregation of negative semidefiniteness (usually shown for the Weak Axiom) is more straightforward 
than that of symmetry. Also, a matrix similar to $\mathbb{V}\left[\partial_{y} \phi, \phi\right]$ has been used in this literature (as “increasing dispersion", see Härdle, Hildenbrand and Jerison (1989)).

Finally, there is an issue about what can be learned from data. To continue our discussion at the beginning of this section, in general the underlying function $\phi$ is not identified. One implication is the following: Suppose there are two groups in the population, type 1 and type 2 . Both types could violate rationality, however, it is entirely possible that the average does not violate rationality. As such, our results provide a lower bound on the violations of rationality in a population. A more precise answer could be obtained, if a linear structure would be assumed. However, in this paper we do not take the linearity assumption for granted. Consequently, under our assumptions this is all we can say about consumer rationality, given the data and mean regression tools.

\section{Empirical Implementation}

In this section we discuss all matters pertaining to the empirical implementation: We give first a brief sketch of the econometrics methods, an overview of the data, mention some issues regarding the econometric methods, and present the results.

\subsection{Econometric Specifications and Methods}

From our identification results in proposition 2.2 and 2.3, we are able to characterize testable implications on nonparametric mean regressions. It is imperative to note that these mean regressions are only the reduced form model, and not a specification of the structural model. As discussed above, these empirically estimable objects are local averages. Consequently, they depend on the position, and we will evaluate them at a set of representative positions (actually 
random draws from the sample). As a characterization of our empirical results, we will give the percentage of positions at which we reject the null. Needless to mention, this may both under- and overestimate the true proportion of violations. In the one case the average may by accident behave nicely, while all individuals behave irrational, while in the other the average may not confirm with rationality, while it is only relatively few nonrational individuals that cause this violation. However, since we are evaluating the population at a large number of local averages over relatively small (but potentially overlapping) neighborhoods, we may expect both effects to be of secondary importance and to balance to some degree. In the spirit of our above results, we could argue that the percentage of rejections obtained using the best approximations given the data is itself at least a good approximation to the underlying fraction of irrational individuals, given the information to our disposal.

As the above discussion should have made clear, the precise testable implications will depend on the independence assumption that a researcher deems realistic. In the demand literature, it is log total expenditure that is taken to be endogenous, and labor income is taken as additional instrument, see Lewbel (1999). The basic reason is preference endogeneity: since the broad aggregates of goods typically considered (e.g., "Food") explain much of total expenditure, it is suspected that the preferences that determine demand for these broad categories of goods, and those how determine total nondurable consumption are dependent. Prices, in turn are assumed to be exogenous, because unlike in IO approaches were the demands for individual goods is analyzed, and price endogeneities may be suspected on grounds that firms charge different consumers differently or that there be unobserved quality differences, the broad categories of good typically analyzed are invariant to these types of considerations. Still, one may question this exogeneity assumption, and we can only emphasize that nothing in our chain of argumentation precludes handling this endogeneity in precisely the same way than total expenditure 
endogeneity.

For purpose of recovering $U$ we have to specify the equation relating the endogenous regressors total expenditure $S$ to instruments only. In this paper, we have settled for the general nonparametric form $Y=\mu(S, Z)=\psi(S, Q)+\sigma_{2}(S, Q) U$, with the normalization $\mathbb{E}[U \mid S, Q]=0$ and $\mathbb{V}[U \mid S, Q]=1$. In supplementary material that may be downloaded from the author's webpage, we discuss issues in the implementation of estimators and test statistics in detail. Here we just mention briefly that we estimate all regression functions by local polynomials, and form pointwise nonparametric tests using these estimators. We evaluate all tests at a grid of $n=3000$ observations that are $i i d$ draws from the data. To derive the asymptotic distribution of our test statistic at each of these observations, we apply bootstrap procedures. In the case of testing homogeneity, we use the fact that the hypothesis, i.e. $m(P, Y, Z)=m(P+\lambda, Y+\lambda, Z)$ so that the null hypothesis may be reformulated by taking derivatives as

$$
D_{p} m\left(\zeta_{0}\right) \iota+\partial_{y} m\left(\zeta_{0}\right)=0 \text { for all } \zeta_{0} \in \operatorname{supp}(P, Y, Z)
$$

where we let $\zeta_{0}=\left(p_{0}, y_{0}, z_{0}\right)$, and analogously for the endogeneous case. This hypothesis is easily rewritten as $R \theta\left(\zeta_{0}\right)=0$, where $R$ denotes the $L-1 \times(L-1)(L+K+2)$ matrix $R=I_{L-1} \otimes\left[\begin{array}{ccc}0 & \iota_{L+1} & 0_{K}\end{array}\right]$, and $\theta\left(\zeta_{0}\right)$ is the vector of stacked levels $m\left(\zeta_{0}\right)$ and $h$ scaled derivatives, $\left(h D_{p} m\left(\zeta_{0}\right), h \partial_{y} m\left(\zeta_{0}\right), h D_{z} m\left(\zeta_{0}\right)\right)$. Next, suppose that $\hat{\theta}\left(\zeta_{0}\right)$ denotes a local polynomial estimator of $\theta\left(\zeta_{0}\right)$ with generated regressors, defined in detail in the econometric supplement to this paper. In addition, assume again a multiplicative heteroscedastic error structure, i.e., $W_{i}-m\left(P_{i}, Y_{i}, Z_{i}\right)=\Sigma_{1}\left(P_{i}, Y_{i}, Z_{i}\right) \eta_{i}$. A natural test statistic is then:

$$
\widehat{\Psi}_{H o m, E x}\left(\zeta_{0}\right)=\left[R\left[\hat{\theta}\left(\zeta_{0}\right)-h^{2} \widehat{\operatorname{bias}}\left(\zeta_{0}\right)\right]\right]^{\prime}\left[R^{\prime}\left[\widehat{\Sigma_{1} \Sigma_{1}^{\prime}}\left(\zeta_{0}\right) \otimes \hat{A}\left(\zeta_{0}\right)\right] R\right]^{-1} R\left[\hat{\theta}\left(\zeta_{0}\right)-h^{2} \widehat{\operatorname{bias}}\left(\zeta_{0}\right)\right],
$$

where $\hat{A}\left(\zeta_{0}\right)=\hat{f}_{X}\left(\zeta_{0}\right)^{-1} B^{-1} C B^{-1}, B$ and $C$ are matrices of kernel constants, and $\widehat{\operatorname{bias}}\left(\zeta_{0}\right)$ 
is a pre-estimator for the bias $^{5}$. Then, by a trivial corollary to the large sample theory of local polynomial estimators with generated regressors found in the supplementary material, $\widehat{\Psi}_{H o m, E x} \stackrel{d}{\rightarrow} \chi_{L-1}^{2}$. This test can thought of as being based on the confidence interval at a certain point, and is a standard pointwise test of derivatives modified by the fact that we consider now systems of equations with generated regressors. Constructing a bootstrap sample under the null, i.e., with homogeneity imposed, is straightforward by simply subtracting log total expenditure from all the log prices and omitting the log total expenditure regressor from the regression (this imposes homogeneity on the regression). Adding the bootstrap residuals $\varepsilon_{i}^{*}$ to the homogeneity imposed regression produces the bootstrap sample $\left(Y_{i}^{*}, X_{i}^{*}\right)=\left(Y_{i}^{*}, X_{i}\right)$. The limiting distribution, as well as arguments showing the consistency of the bootstrap, are then straightforward from the asymptotics of local polynomials.

The case of testing symmetry is more involved. To derive a test for the first case, stack the $1 / 2 L(L-1)$ nonlinear restrictions $R_{k l}\left(\theta, \zeta_{0}\right)$, at a fixed position $x_{0}$,

$R_{k l}\left(\theta, \zeta_{0}\right)=\partial_{p_{k}} m^{l}\left(\zeta_{0}\right)+\partial_{y} m^{l}\left(\zeta_{0}\right) m^{k}\left(\zeta_{0}\right)-\left(\partial_{p_{l}} m^{k}\left(\zeta_{0}\right)+\partial_{y} m^{k}\left(\zeta_{0}\right) m^{l}\left(\zeta_{0}\right)\right)=0, \quad k, l=1, . ., L-1, k>l$,

into a vector $R$. Consequently, " $D_{p} m\left(\zeta_{0}\right)+\partial_{y} m\left(\zeta_{0}\right) m\left(\zeta_{0}\right)^{\prime}$ symmetric for all $\zeta_{0} \in \operatorname{supp}(P, Y, Z)$ " becomes " $R\left(\theta, \zeta_{0}\right)=0$ for all $\zeta_{0} \in \operatorname{supp}(P, Y, Z)$ ". This suggest using the quadratic form

$$
\widehat{\Gamma}_{S y m, E x}\left(\zeta_{0}\right)=\left[R\left(\hat{\theta}, \zeta_{0}\right)\right]^{\prime} R\left(\hat{\theta}, \zeta_{0}\right)
$$

and check whether this is significantly bigger than zero. Again, building upon the large sample theory of local polynomial estimators with generated regressors, we could derive the the limiting distribution. However, this is even more involved as before, and the bootstrap appears to be the method of choice. An adaptation of the idea of Haag, Hoderlein and Pendakur (2007)

\footnotetext{
${ }^{5}$ Since the bias contains largely second derivatives, we may use a local quadratic or cubic estimator for the second derivative, with a substantial amount of undersmoothing.
} 
for deriving a bootstrap version of the test statistic allows to determine an estimator for the true distribution of the test statistic under the null (by exploiting the structure of the Kernel estimator, as well as the fact that under the null the bias vanishes). This has the added benefit that the consistency of the bootstrap may be established along similar lines as in Haag, Hoderlein and Pendakur (2007).

Finally, a bootstrap procedure for testing negative semidefiniteness may be devised using a similar idea by Härdle and Hart (1993). Essentially, the idea is to look at the bootstrap distribution of the largest eigenvalue. This procedure is consistent, provided there is no multiplicity of eigenvalues, which is not a problem in our application. For technical details we refer again to the supplementary material that may be downloaded from the author's webpage.

\subsection{Data}

The FES reports a yearly cross section of labor income, expenditures, demographic composition and other characteristics of about 7,000 households. We use the years 1974-1993, but exclude the respective Christmas periods as they contain too much irregular behavior. As already mentioned above, we focus on two types of subpopulations, one person and two person households. Specifically, in the one person household category we consider all single person households, but we also stratify according to men and women. In te category of two person households we narrow the subpopulation down a bit further to include households where both are adults and at least one is working. This is going to be our benchmark subpopulation, because it was the one commonly used in the parametric demand system literature, see Lewbel (1999). We provide several tables showing various summary statistics of our data in the appendix.

The expenditures of all goods are grouped into three categories. The first category is related to food consumption and consists of the subcategories food bought, food out (catering) and 
tobacco, which are self explanatory. The second category contains expenditures which are related to the house, namely housing (a more heterogeneous category; it consists of rent or mortgage payments) and household goods and services. Finally, the last group consists of motoring and fuel expenditures, categories that are often related to energy prices. For brevity, we call these categories food, housing and energy. These broader categories are formed since more detailed accounts suffer from infrequent purchases (recall that the recording period is 14 days) and are thus often underreported. Together they account for approximately half of total expenditure on average, leaving a large forth residual category. We removed outliers by excluding the upper and lower $2.5 \%$ of the population in the three groups.

Labor income is constructed as in the definition of household below average income study (HBAI). It is roughly defined as net labor income after taxes, but including state transfers. We employ the remaining household covariates as regressors, including a time trend in the set of covariates. We use principal components to reduce this larger vector $Q$ to some three orthogonal approximately continuous components, mainly because we require continuous covariates for nonparametric estimation. While this has some additional advantages, it is arguably ad hoc. However, we performed some robustness checks like alternating the components or adding parametric indices to the regressions or including separately a monthly time trend, and the results do not change in an appreciable fashion.

Finally, the prices come from an aggregate time series, the Retail Price Index which is published at the National Statistics Online web site. It is monthly data spanning all twenty years we consider. As an aside, the FES was collected to construct exactly these price indices. This completes our list of variables. 


\subsection{Empirical Results in Detail}

\subsubsection{Result for the Benchmark Group: Two Person Households}

We first start out with our benchmark group, i.e., two person households, both of which are working (from now on "couples"). As already mentioned, this subpopulation has often been selected in demand application using the FES, because the couples are believed to be relatively homogenous and exhibit less measurement error.

Although they are not in the focus of this paper, because they are building blocks for our test statistics we display in the appendix some nonparametric estimates of the function and the derivatives for this subpopulation. In figures 1-3 in the appendix we show the budget shares of the three categories of goods against log total expenditure. Note that the food and energy budget shares are everywhere downward sloping in total expenditure, whereas housing is only weakly de- or increasing, but in absolute value relatively constant across the expenditure range. This conforms well with results obtained elsewhere in the literature, see Lewbel (1999). Moreover, we also show compensated own price, as well as compensated cross price effects of the symmetrized Slutsky matrix in figures 4 - 9. The figures show that the compensated own price effects of food, energy and housing are negative, as predicted by theory. The cross price effects are smaller (in absolute value) in comparison, but note the relatively large confidence bands around all the derivatives, which arise because of our unrestrictive specification. The negative and dominant own price effects combined with the large standard errors are indicative that negative semidefiniteness may not be rejected. All functions are plotted at the mean level of all other regressors, and obviously at other values of the regressors the picture varies. The largely negative compensated own price effects, however, remain preserved.

Turning to the test statistics, they are constructed such that the implications of economic 
theory are true under the null. Moreover, the tests are to be performed pointwise at the individual observations. A rejection at an individual observation means that the original condition, e.g., negative semidefiniteness in the underlying heterogeneous population, cannot hold in the "neighborhood" of an individual. Although this gives an accurate picture of the behavior on individual level, it results in a flood of results, and we aggregate across the population as a means of condensing the result. Hence, our interest centers on the proportion of the population, for which a certain property is not violated.

Before discussing the results in detail, two important issues have to be clarified. The first concerns the functional form. Using the test of Haag, Hoderlein and Mihaleva (2006), we reject the null that the regression is a Quadratic Almost Ideal. More precisely, the value of the test statistic is 7.32 , while the 0.95 quantile of the bootstrap distribution is 3.65 . Indeed, this finding strongly suggests using a flexible nonparametric form - otherwise rejections of economic hypotheses may occur just because we have chosen the wrong functional form.

The second issue is endogeneity: We use total expenditure as regressor, which is potentially endogenous. Employing again a Haag, Hoderlein and Mihaleva (2006) type test, we reject the hypothesis that $U$ should be excluded from the regression with a $p$-value of 0.01 . Although this strongly suggests that a control function correction for endogeneity should be adopted, below we will see that in terms of economic restrictions the results are largely comparable.

The main results are condensed in tables 3.1 to 3.3. We report the results using total expenditure under both exogeneity and endogeneity, where we handle the latter by including control function residuals. The results using the instruments plus controls regressions produce similar results to the standard endogeneity correction, and are therefore not reported here.

Table 3.1 shows results for tests of the test of the homogeneity hypothesis. 


\begin{tabular}{l||ll} 
Hypothesis & 0.90 & 0.95 \\
\hline \hline Homogeneity under Exogeneity & 0.84 & 0.94 \\
Homogeneity under Endogeneity & 0.86 & 0.97
\end{tabular}

Table 3.1. Percentage of Two Person Households in Accordance with Homogeneity

The table shows in the first column again the hypothesis that is being tested, while the second and third row display the number of non rejections at the 0.90 and 0.95 confidence level. From these results it is obvious that homogeneity is well accepted. The results improve slightly if we move to a scenario of endogeneity, but this was to be expected as we also add one regressor (the control function residuals), and hence the standard errors become larger. With respect to the violations, no clear pattern of violations emerges, i.e. no clustering at certain household characteristics. Overall, the results are so good that we will now report results were we impose homogeneity.

Next, the results for symmetry are displayed in table 3.2. As before, the percentage of non rejections at the significance levels 0.90 and 0.95 are displayed.

\begin{tabular}{l||cc} 
Hypothesis & 0.90 & 0.95 \\
\hline \hline Symmetry under Exogeneity & 0.70 & 0.77 \\
Symmetry under Exogeneity and Homogeneity & 0.84 & 0.90 \\
Symmetry under Endogeneity and Homogeneity & 0.84 & 0.91
\end{tabular}

Table 3.2. Percentage of Two Person Households in Accordance with Slutsky Symmetry

Observe that we first impose homogeneity, and then correct for endogeneity. Imposing homogeneity is justified because it is hard to imagine circumstances under which symmetry (or negative semidefiniteness) holds, while homogeneity does not, and we have already seen that 
homogeneity is likely to hold across the population. Accounting for homogeneity improves the result substantially, which is particularly comforting as the introduction of homogeneity actually reduces the number of regressors, i.e., the standard errors become smaller. The inclusion of endogeneity improves the result marginally, but this was to be expected because of the increase in standard errors. We could not find any obvious structure in the remaining rejections.

Finally, consider the negative semidefiniteness hypothesis. We incorporate the results of the symmetry test by considering this property for the symmetrized Slutsky matrix. With respect to the individual results we report both the uncompensated and compensated price effect (i.e., Slutsky) matrices. The percentage of the population for which negative semidefiniteness can not be rejected at the 0.95 percent confidence level are as follows:

Hypothesis

\begin{tabular}{l||lc}
\hline \hline Negative Semidefiniteness under Exogeneity & 0.77 & 0.69 \\
Negative Semidefiniteness under Exogeneity and Homogeneity & 0.79 & 0.81 \\
Negative Semidefiniteness under Endogeneity and Homogeneity & 0.80 & 0.83
\end{tabular}

Table 3.3. Percentage of Two Person Households in Accordance with Negative Semidefiniteness

Note from the first row the somewhat unexpected result that the uncompensated price effect matrix is more often negative semidefinite than the compensated one. This seems to disagree with economic theory. However, a careful examination of the individual results shows that matrices that were only barely negative semidefinite get slightly perturbed by the relatively strong income effect matrix (i.e., the difference between the two matrices).

Moreover, this curiosity disappears once we impose the almost uniformly accepted zero homogeneity property. Imposing homogeneity, the Slutsky matrix is now more often negative 
semidefinite than the uncompensated price effect matrix, which is particularly comforting as the introduction of homogeneity actually reduces the number of regressors, i.e., the standard errors become smaller. Finally, the inclusion of endogeneity improves the result even further, but this was again to be expected because of the increase in standard errors As before, there is no obvious structure in the remaining rejections.

Finally, Endogeneity generally seems to play a minor role in this application, which disagrees with some findings in the literature which use the same data, in particular Blundell, Chen and Kristensen (2007).

In summary, these results establish that our quite general nonparametric approach works well in data sets of the size typically found in applications, and that it corroborates economic theory. As the main econometric caveat for our analysis, the following point should nevertheless be mentioned: Indeed, some point estimates of the Slutsky matrix appear to be not symmetric. The same is, perhaps even to a larger extent, also true of negative semidefiniteness. However, it is especially the cross price effects that are measured with very low precision, and hence have huge standard errors. This familiar problem of parametric demand analysis (cf. Lewbel (1999)) is aggravated by the high dimensional nonparametric approach taken here. Hence, we should stress the fact that we were not able to reject these hypotheses. However, we want to emphasize squarely that this is inevitable given the generality of our assumptions: If we are not willing to assume more, this is what we obtain. While we can safely rule out mistakes by wrongly assuming a certain functional form or too much homogeneity across individuals, the lower precision is the price to pay. A logical next step would be to carefully introduce semiparametric structure, but we leave this step for future research. Nevertheless, it is noteworthy that overall economic theory provides an acceptable hypothesis for the population, as is indicated by results beyond the $80 \%$ mark. Hence, at least in this specific subpopulation, economic theory fares well. 
That the acceptance of rationality is less than $100 \%$ in this subpopulation my be attributed to a number of factors. One of them is that the data are collected on household level, while rationality restrictions pertain to individuals. The passage from individual decisions to those of the households is less than trivial, as has recently been emphasized (see Browning and Chiappori (1998), Fortin and Lacroix (1997) and Cherchye and Vermeulen (2008), amongst others). Hence we are now going to consider one person households and compare them with the composite ones we have just analyzed.

\subsubsection{Comparison of Two Person with Single Households}

This subsection considers the behavior of the same tests, involving the same nonparametric building blocks, as well as the FES data, but now focussing on single households. Economic theory only makes statements about individual behavior; in light of this, the fact that we have treated two person households like individuals may seem to be problematic. To give a particularly simple example, if one person solely decides about buying bundles of goods under one price regime, while the other does so under another price regime, there is no reason why even basic rationality requirements like the Weak Axiom of Revealed Preference were to hold for the composite household. More generally, we face a problem of aggregation of preferences, and this problem has spurned extensive research, see Browning and Chiappori (1998), Fortin and Lacroix (1997) and Cherchye and Vermeulen (2008). An upshot of this literature is that we would expect single households to perform better than couples when it comes to obeying rationality restrictions. The following results, however, show a less clear cut picture. We report the results separately for men (tables 3.4-3.6) and women (tables (3.7-3.9), but we have also pooled both groups without obtaining materially different results. Summary statistics for all subpopulations can be found in the appendix. 
We start out with the population of single men, and we consider again homogeneity first, because it is a basic requirement for the following analysis. We obtain similarly high acceptance rates as before, which is comforting.

\begin{tabular}{l||ll} 
Hypothesis & 0.90 & 0.95 \\
\hline \hline Homogeneity under Exogeneity & 0.89 & 0.94 \\
Homogeneity under Endogeneity & 0.90 & 0.95
\end{tabular}

Table 3.4. Percentage of Single Males in Accordance with Homogeneity

Note also, that we do not find a lot of difference with respect to the correction of endogeneity; the overall result is qualitatively similar to two person households with respect to homogeneity. Next, we turn to symmetry of the Slutsky matrix. The results are again summarized in the following table:

\begin{tabular}{l||cc} 
Hypothesis & 0.90 & 0.95 \\
\hline \hline Symmetry under Exogeneity & 0.72 & 0.82 \\
Symmetry under Exogeneity and Homogeneity & 0.80 & 0.88 \\
Symmetry under Endogeneity and Homogeneity & 0.77 & 0.86
\end{tabular}

Table 3.5. Percentage of Single Males in Accordance with Slutsky Symmetry

Again the results are very similar - if marginally worse - than for couples, but observe that the results actually get worse when endogeneity is corrected for. Since the correction for endogeneity actually involves the introduction of control functions, and hence would result in tests of lower power due to the increase in dimensionality of the regressions, the fact that the number of rejections of rationality increases casts doubts on the specific correction for endogeneity. This conclusion gets corroborated by the results with respect to negative semidefiniteness, which are displayed in table 3.6: 


\begin{tabular}{l||c}
\hline \hline Negative Semidefiniteness under Exogeneity & 0.76 \\
Negative Semidefiniteness under Exogeneity and Homogeneity & 0.81 \\
Negative Semidefiniteness under Endogeneity and Homogeneity & 0.82
\end{tabular}

Table 3.6. Percentage of Single Males in Accordance with Slutsky Negative Semidefiniteness

We again find virtually unchanged results compared with couples. The results do not change qualitatively when we consider single females, see the next three tables (3.7-3.9): Roughly speaking, homogeneity fares a bit worse, while in particular the fraction in accordance with negative semidefiniteness is substantially higher.

\begin{tabular}{l||cc} 
Hypothesis & 0.90 & 0.95 \\
\hline \hline Homogeneity under Exogeneity & 0.79 & 0.89 \\
Homogeneity under Endogeneity & 0.79 & 0.90
\end{tabular}

Table 3.7. Percentage of Single Females in Accordance with Homogeneity

These results are somewhat worse than the corresponding numbers for males. The same is also true for symmetry under exogeneity, but once homogeneity is imposed, the results become very comparable (table 3.8).

\begin{tabular}{l||cc} 
Hypothesis & 0.90 & 0.95 \\
\hline \hline Symmetry under Exogeneity & 0.60 & 0.69 \\
Symmetry under Exogeneity and Homogeneity & 0.80 & 0.90 \\
Symmetry under Endogeneity and Homogeneity & 0.78 & 0.87
\end{tabular}

Table 3.8. Percentage of Single Females in Accordance with Slutsky Symmetry

Finally, with respect to negative semidefiniteness of the Slutsky matrix, single women outper- 
form both their male counterparts, as well as couples. This may be seen as a hint that at least the female part of the Singles population exhibits more rational behavior.

\begin{tabular}{l||l} 
Hypothesis & 0.89 \\
\hline \hline Negative Semidefiniteness under Exogeneity & 0.90 \\
Negative Semidefiniteness under Exogeneity and Homogeneity & 0.90
\end{tabular}

Table 3.9. Percentage of Single Females in Accordance with Negative Semidefiniteness

The results do not change in any material way when we pool men and women to one "single" category; if anything the results improve slightly compared to each category in isolation. However, the overall fluctuations between all the various subpopulations are relatively minor and do not show any clear pattern. Hence we draw the conclusion that the rationality restrictions are well, but not perfectly accepted in all subpopulations. At this point we would like to emphasize that there may be issues of measurement error with single households, in particular, it is quite possible that they form parts of extended households living at several locations, and/or are their consumption behavior is partially determined by institutions, in particular their workplaces. As such we also want to express our scepticism whether $100 \%$ compliance with rationality is ever to be expected. In summary, the conclusion we draw from our comparison is that it is hard to detect a material difference between collective decision makers and individuals in our data, but we acknowledge that this may be due to limitations in our data.

\section{Conclusion}

In this paper we have established that it is really only necessary to impose one substantial identifying restriction in order to perform most of demand analysis empirically, i.e., the conditional 
independence assumption $A 2.2$. Once this assumption is in place (which, as has been pointed out, may be challenging at times to verify), nothing else apart from regularity conditions has to be imposed to test conclusively the main elements of demand theory, in particular homogeneity and negative semidefiniteness. It is a key feature of our approach that no other material assumption has to be imposed. This is particularly true for functional form or population homogeneity assumptions.

While this is true without any qualifiaction for Homogeneity and Negative Semidefiniteness of the Sltusky Matrix, Symmetry of the Slutsky matrix turns out to be the only major implication of rationality that will only ever be testable under additional identification assumptions. The doubts on the empirical verifiability of this property suggests that economic theory should perhaps concentrate on a model of demand based on the Weak Axiom, such as in Kihlstrom, Mas-Colell and Sonnenschein (1976) or Quah (2000), and that is entirely testable according to our model.

The conditional independence assumption plays a critical role in this paper. It may have quite complex economic implications, and may be rightfully questioned in some applications. In our application the potentially questionable part was the implicit assumption about stability of the conditional distribution of preferences over medium time horizons. Hence, much as in the older parametric literature, the credibility of the results hinges on the quality of the data. However, the assumption is sufficiently general to nest a variety of scenarios including control function IV, and proxy variables, which is not detailed in this paper but straightforward. The main task of the researcher is to specify the precise form of the independence assumptions that he believes to be most realistic on economic grounds. This paper has established that from this starting point on empirical economic analysis can proceed without any major additional restriction. In our application we exemplify how this can be done, and discuss evidence about 
the empirical extend of the difference in consumer behavior between collective households and individuals. We would like to point out that our approach allowed us to focus on this question without additional assumptions, and we hope that this example encourages future applications using a similar framework.

Acknowledegements: We have received helpful comments from two anonymous referees, and from the editor Peter Robinson, as well as from Richard Blundell, Andrew Chesher, Joel Horowitz, Arthur Lewbel, Rosa Matzkin, Whitney Newey and from seminar participants at Berkeley, Brown, ESEM, Copenhagen, Madrid, Northwestern, LSE, Stanford, Tilburg, UCL and Yale. The usual disclaimer applies. I am also indebted to Sonya Mihaleva for excellent research assistance.

\section{Appendix}

\section{A.1 Assumption 2.3: Regularity Conditions}

Assume that the demand functions $\phi$ be continuously differentiable in $x$ for all $x \in \mathcal{X} \subseteq$ $\mathbb{R}^{L+1}$. This restricts preferences to be continuous, strictly convex and locally nonsatiated, with associated utility functions everywhere twice differentiable. Assume in addition that $\mu$ be continuously differentiable in $s$ for all $s \in \mathcal{S} \subseteq \mathbb{R}^{K+1}$, and that $D_{s} \mu$ has full column rank almost surely. Assume that preferences be additively separable over time, which justifies the use of total expenditure as wealth. Moreover, we confine ourselves to observationally distinct preferences, i.e. if $v_{j}, v_{k} \in \mathcal{V}$ and $v_{j} \neq v_{k}$, then there exists a set $\mathcal{X} \subseteq \mathbb{R}^{L+1}$ with $\mathbb{P}(\mathcal{X})>0$, such that $\forall x \in X: D_{x} \phi\left(x, v_{1}\right) \neq D_{x} \phi\left(x, v_{2}\right)$. Finally, we require the following condition for dominated convergence: there exists a function $g$, s. th. $\left\|v e c\left[D_{x} \phi(x, \vartheta(q, a))\right]\right\| \leq g(a)$, with 
$\int g(a) F_{A}(d a)<\infty$, uniformly in $(x, q)$.

\section{A.2 Proofs of Lemmata and Propositions}

\section{Proof of Lemma 2.1:}

First recall that, by definition, $0 \leq W \leq 1$. Thus, the expectation exists and $\mathbb{E}[|W|] \leq c<\infty$, where $c$ is a generic constant (the same holds for the second moment). From this it follows that all conditional expectations exist as well, and are bounded.

Next, let $x, z$ be fixed, but arbitrary.

$$
\begin{aligned}
D_{x} m(x, q, u) & =D_{x} \int_{\mathcal{A}} \phi(x, \vartheta(q, a)) F_{A \mid X, Q, U}(d a, x, q, u) \\
& =D_{x} \int_{\mathcal{A}} \phi(x, \vartheta(q, a)) F_{A \mid Q, U}(d a, q, u),
\end{aligned}
$$

due to $A 2.2 \Longrightarrow F_{A \mid X, Q, U}=F_{A \mid Q, U}$. Using dominated convergence, we obtain that the rhs equals

$$
\int_{\mathcal{A}} D_{x} \phi(x, \vartheta(q, a)) F_{A \mid Q, U}(d a, q, u)
$$

But due to $A 2.2$ this is (a version of) $\mathbb{E}\left[D_{x} \phi \mid X=x, Z=z\right]$. Upon inserting random variables for the fixed $z, y$ the statement follows. For $(i i)$, by the same arguments $D_{s} M(s, z)=\mathbb{E}\left[D_{x} \phi \mid S=\right.$ $s, Z=z] D_{s} \mu(s, z)$. Postmultiplying with $D_{s} \mu(s, z)^{-}$produces the result.

Proof of Proposition 2.2: Adding up follows trivially by taking conditional expectations of $\iota^{\prime} \phi=1$ (a.s). To see homogeneity, recall that

$$
m(x+\lambda, q, u)=\int_{\mathcal{A}} \phi(x+\lambda, \vartheta(a, q)) F_{A \mid X, Q, U}(d a, x+\lambda, q, u)
$$

Inserting $\phi(x+\lambda, v)=\phi(x, v)$ as well as $F_{A \mid X, Q, U}(a, x+\lambda, q, u)=F_{A \mid X, Q, U}(a, x, q, u)$ produces

$$
\int_{\mathcal{A}} \phi(x+\lambda, \vartheta(a, q)) F_{A \mid X, Q, U}(d a, x+\lambda, q, u)=\int_{\mathcal{A}} \phi(x, \vartheta(a, q)) F_{A \mid X, Q, U}(d a, x, q, u)=m(x, q, u) .
$$


The same argumentation holds also for

$$
M(s+\lambda, q, u)=\int_{\mathcal{A}} \phi(\mu(s+\lambda, q, u)+\lambda, \vartheta(a, q)) F_{A \mid S, Q, U}(d a, s, q, u),
$$

using additionally that $\mu(s+\lambda, q, u)=\mu(s, q, u)$. Finally, the statements about the derivatives follow straightforwardly from the fact that homogeneity implies that $D_{p} \phi \iota+\partial_{y} \phi=0$ (a.s.), in connection with Lemma 2.1.

Q.E.D.

Proof of Proposition 2.3: Ad Negative Semidefiniteness : Let $A(\omega), \omega \in \Omega$, denote any random matrix. If $p^{\prime} A(\omega) p \leq 0$ for all $\omega \in \Omega$ and all $p \in \mathbb{R}^{L}$, then, upon taking expectations w.r.t. an arbitrary probability measure $F$, it follows that

$$
\int p^{\prime} A(\omega) p F(d \omega) \leq 0 \Leftrightarrow p^{\prime} \int A(\omega) F(d \omega) p \leq 0, \text { for all } p \in \mathbb{R}^{L}
$$

From this $\mathfrak{S}$ nsd (a.s.) $\Rightarrow \mathbb{E}[\mathfrak{S} \mid X, Z]$ nsd (a.s.) is immediate. Let $\mathbb{E}[\mathfrak{S} \mid X, Z]=B$, and note that since the definition of negative semidefiniteness of a square matrix $B$ of $\operatorname{dim} L$ involves the quadratic form, $p^{\prime} B p \leq 0$, we see that if we put $\bar{B}=B+B^{\prime}$, we have

$$
p^{\prime} \bar{B} p=2 p^{\prime} B p \text { for all } p \in \mathbb{R}^{L}
$$

and $\bar{B}$ symmetric, implying that $B$ is negative semidefinite if and only if $\bar{B}$ is negative semidefinite. From

$$
\begin{aligned}
B & =\mathbb{E}[\mathfrak{S} \mid X, Z] \\
& =\mathbb{E}\left[D_{p} \phi \mid X, Z\right]+\mathbb{E}\left[\partial_{y} \phi \phi^{\prime} \mid X, Z\right]+\mathbb{E}\left[\phi \phi^{\prime} \mid X, Z\right]-\mathbb{E}[\operatorname{diag}(\phi) \mid X, Z] \\
& =B_{1}+B_{2}+B_{3}+B_{4}
\end{aligned}
$$

follows that $\bar{B}=B+B^{\prime}=B_{1}+B_{2}+B_{3}+B_{4}+B_{1}^{\prime}+B_{2}^{\prime}+B_{3}^{\prime}+B_{4}^{\prime}=\bar{B}_{1}+\bar{B}_{2}+2\left(B_{3}+B_{4}\right)$, since $B_{3}$ and $B_{4}$ are symmetric. Thus we have that

$$
\mathfrak{S} \text { nsd (a.s.) } \Rightarrow \bar{B}_{1}+\bar{B}_{2}+2\left(B_{3}+B_{4}\right) \text { nsd (a.s.) }
$$


From Lemma 2.1 it is apparent that $\bar{B}_{1}=D_{p} m+D_{p} m^{\prime}$. To see that $\bar{B}_{2}=\partial_{y} m_{2}(x, z)$, first note that due to the boundedness of $W$ the second moments and conditional moments exist, so that

$$
\begin{aligned}
\partial_{y} m_{2}(x, z) & =\partial_{y} \int_{\mathcal{A}} \phi(x, \vartheta(q, a)) \phi^{\prime}(x, \vartheta(q, a)) F_{A \mid X, Z}(d a ; x, z) \\
& =\mathbb{E}\left[\partial_{y}\left(\phi \phi^{\prime}\right) \mid X=x, Z=z\right] \\
& =\mathbb{E}\left[\partial_{y} \phi \phi^{\prime}+\phi \partial_{y} \phi^{\prime} \mid X=x, Z=z\right]=\bar{B}_{2}
\end{aligned}
$$

$B_{3}$ and $B_{4}$ are trivial. Upon inserting random variables, the statement follows. The proof using the regression with instruments and controls follows by the same arguments in connection with Lemma 2.1 (iii).

Q.E.D.

Ad Symmetry To see the "if" direction, note first that $\mathfrak{S}$ symmetric iff $K=D_{p} \phi+\partial_{y} \phi \phi^{\prime}$ is symmetric, which implies that $\mathbb{E}[K \mid Y, Z]$ is symmetric since $A_{i j}=\mathbb{E}\left[K_{i j} \mid Y, Z\right]=\mathbb{E}\left[K_{j i} \mid Y, Z\right]=$ $A_{j i}$, where the subscript $i j$ denotes the $i j$-th element of the matrix. This implies in turn that

$$
\begin{aligned}
\mathbb{E}[K \mid Y, Z] & =\mathbb{E}\left[D_{p} \phi \mid Y, Z\right]+\mathbb{E}\left[\partial_{y} \phi \phi^{\prime} \mid Y, Z\right] \\
& =\mathbb{E}\left[D_{p} \phi \mid Y, Z\right]+\mathbb{E}\left[\partial_{y} \phi \mid Y, Z\right] \mathbb{E}\left[\phi^{\prime} \mid Y, Z\right]+\mathbb{V}\left[\partial_{y} \phi, \phi \mid Y, Z\right]
\end{aligned}
$$

is symmetric, from which $\mathbb{E}\left[D_{p} \phi \mid Y, Z\right]+\mathbb{E}\left[\partial_{y} \phi \mid Y, Z\right] \mathbb{E}\left[\phi^{\prime} \mid Y, Z\right]$ is symmetric if $\mathbb{V}\left[\partial_{y} \phi, \phi \mid Y, Z\right]$ is assumed to be symmetric. By Lemma 2.1. this equals $D_{p} m+\partial_{y} m m^{\prime}$.

To establish the "only if" direction, we have to show that $\mathbb{V}\left[\partial_{y} \phi, \phi \mid Y, Z\right]$ not symmetric implies that $\mathfrak{S}$ symmetric does not imply that $D_{p} m+\partial_{y} m m^{\prime}$ be symmetric. To this end, assume again that $\mathfrak{S}$ be symmetric, and consider (A.2). In this case, $\mathbb{E}[K \mid Y, Z]$ is symmetric, but due to $\mathbb{V}\left[\partial_{y} \phi, \phi \mid Y, Z\right]$ not symmetric we obtain that

$$
D_{p} m+\partial_{y} m m^{\prime}=\mathbb{E}[K \mid Y, Z]-\mathbb{V}\left[\partial_{y} \phi, \phi \mid Y, Z\right]
$$

has to be not symmetric as well.

Q.E.D. 


\section{Appendix 2}

Summary Statistics of Data: Household Characteristics, Income and Budget Shares after Outlier Removal

\section{Couples}

\begin{tabular}{|c|c|c|c|c|c|c|}
\hline Variable & Minimum & 1st Quartile & Median & Mean & 3rd Quartile & maximum \\
\hline number of female & 1 & 1 & 1 & 1.001 & 1 & 2 \\
\hline number of retired & 0 & 0 & 0 & 0.046 & 0 & 1 \\
\hline number of earners & 1 & 1 & 2 & 1.749 & 2 & 2 \\
\hline Age of HHhead & 19 & 30 & 47 & 45 & 57 & 86 \\
\hline Fridge & 0 & 1 & 1 & 0.995 & 1 & 1 \\
\hline Washing Machine & 0 & 1 & 1 & 0.887 & 1 & 1 \\
\hline Centr. Heating & 0 & 1 & 1 & 0.829 & 1 & 1 \\
\hline TV & 0 & 1 & 1 & 0.877 & 1 & 1 \\
\hline Video & 0 & 0 & 0 & 0.377 & 1 & 1 \\
\hline $\mathrm{PC}$ & 0 & 0 & 0 & 0.061 & 0 & 1 \\
\hline number of cars & 0 & 1 & 1 & 1.315 & 2 & 4 \\
\hline number of rooms & 1 & 5 & 5 & 5.363 & 6 & 15 \\
\hline ln.HHincome & 3.818 & 4.802 & 5.314 & 5.289 & 5.805 & 6.602 \\
\hline BS GROUP 1 & 0.048 & 0.115 & 0.158 & 0.166 & 0.209 & 0.349 \\
\hline Food bought & 0.004 & 0.092 & 0.135 & 0.148 & 0.189 & 0.591 \\
\hline Catering & 0 & 0.019 & 0.041 & 0.049 & 0.069 & 0.277 \\
\hline Tobacco & 0 & 0 & 0 & 0.018 & 0.026 & 0.220 \\
\hline BS GROUP 2 & 0.09 & 0.218 & 0.294 & 0.306 & 0.386 & 0.598 \\
\hline Housing & 0 & 0.122 & 37.195 & 0.207 & 0.280 & 0.582 \\
\hline HHgoods & 0 & 0.021 & 0.033 & 0.043 & 0.089 & 0.643 \\
\hline HHservices & 0 & 0.020 & 0.031 & 0.043 & 0.051 & 0.415 \\
\hline
\end{tabular}




\section{Singles}

\begin{tabular}{|c|c|c|c|c|c|c|}
\hline Variable & Minimum & 1st Quartile & Median & Mean & 3rd Quartile & maximum \\
\hline number of female & 0.0000 & 0.0000 & 1.0000 & 0.6296 & 1.0000 & 1.0000 \\
\hline number of retired & 0.0000 & 0.0000 & 0.0000 & 0.0000 & 0.0000 & 0.0000 \\
\hline number of earners & 1.0000 & 1.0000 & 1.0000 & 1.0000 & 1.0000 & 1.0000 \\
\hline Age of HHhead & 18.0000 & 29.0000 & 38.0000 & 40.6347 & 52.5000 & 88.0000 \\
\hline Fridge & 0.0000 & 1.0000 & 1.0000 & 0.9745 & 1.0000 & 1.0000 \\
\hline Washing Machine & 0.0000 & 0.0000 & 1.0000 & 0.6850 & 1.0000 & 1.0000 \\
\hline Centr.Heating & 0.0000 & 0.0000 & 1.0000 & 0.7292 & 1.0000 & 1.0000 \\
\hline TV & 0.0000 & 1.0000 & 1.0000 & 0.7571 & 1.0000 & 1.0000 \\
\hline Video & 0.0000 & 0.0000 & 0.0000 & 0.4631 & 1.0000 & 1.0000 \\
\hline $\mathrm{PC}$ & 0.0000 & 0.0000 & 0.0000 & 0.0506 & 0.0000 & 1.0000 \\
\hline number of cars & 0.0000 & 0.0000 & 1.0000 & 0.7034 & 1.0000 & 4.0000 \\
\hline number of rooms & 1.0000 & 3.0000 & 4.0000 & 4.3004 & 5.0000 & 11.0000 \\
\hline ln.HHincome & 3.3478 & 4.5103 & 4.9846 & 4.9219 & 5.3841 & 6.0112 \\
\hline BSGROUP1 & 0.0481 & 0.1234 & 0.1725 & 0.1832 & 0.2336 & 0.4041 \\
\hline Food bought & 0.0000 & 0.0634 & 0.1013 & 0.1118 & 0.1485 & 0.3823 \\
\hline Catering & 0.0000 & 0.0177 & 0.0400 & 0.0512 & 0.0723 & 0.2997 \\
\hline Tobacco & 0.0000 & 0.0000 & 0.0000 & 0.0201 & 0.0276 & 0.3863 \\
\hline BSGroup2 & 0.1045 & 0.2637 & 0.3628 & 0.3647 & 0.4596 & 0.6626 \\
\hline Housing & 0.0000 & 0.1645 & 0.2605 & 0.2657 & 0.3555 & 0.6275 \\
\hline HHgoods & 0.0000 & 0.0093 & 0.0281 & 0.0582 & 0.0733 & 0.6811 \\
\hline HHservices & 0.0000 & 0.0263 & 0.0419 & 0.0523 & 0.0635 & 0.4495 \\
\hline BSGROUP3 & 0.0174 & 0.0675 & 0.1327 & 0.1599 & 0.2243 & 0.5048 \\
\hline Motoring & 0.0000 & 0.0000 & 380717 & 0.1029 & 0.1626 & 0.4973 \\
\hline Fuel & 0.0000 & 0.0297 & 0.0458 & 0.0570 & 0.0720 & 0.5048 \\
\hline
\end{tabular}




\section{Single Men}

\begin{tabular}{|c|c|c|c|c|c|c|}
\hline Variable & Minimum & 1st Quartile & Median & Mean & 3rd Quartile & maximum \\
\hline number of retired & 0.0000 & 0.0000 & 0.0000 & 0.0000 & 0.0000 & 0.0000 \\
\hline number of earners & 1.0000 & 1.0000 & 1.0000 & 1.0000 & 1.0000 & 1.0000 \\
\hline Age of HHhead & 19.0000 & 29.0000 & 36.0000 & 38.6878 & 48.0000 & 84.0000 \\
\hline Fridge & 0.0000 & 1.0000 & 1.0000 & 0.9589 & 1.0000 & 1.0000 \\
\hline Washing Machine & 0.0000 & 0.0000 & 1.0000 & 0.6245 & 1.0000 & 1.0000 \\
\hline Centr.Heating & 0.0000 & 0.0000 & 1.0000 & 0.7340 & 1.0000 & 1.0000 \\
\hline $\mathrm{TV}$ & 0.0000 & 0.0000 & 1.0000 & 0.7391 & 1.0000 & 1.0000 \\
\hline Video & 0.0000 & 0.0000 & 0.0000 & 0.4996 & 1.0000 & 1.0000 \\
\hline $\mathrm{PC}$ & 0.0000 & 0.0000 & 0.0000 & 0.0830 & 0.0000 & 1.0000 \\
\hline number of cars & 0.0000 & 0.0000 & 1.0000 & 0.8281 & 1.0000 & 4.0000 \\
\hline number of rooms & 1.0000 & 3.0000 & 4.0000 & 4.2951 & 5.0000 & 11.0000 \\
\hline ln.HHincome & 3.3807 & 4.6823 & 5.1340 & 5.0627 & 5.4860 & 6.2027 \\
\hline BSGROUP1 & 0.0435 & 0.1152 & 0.1653 & 0.1802 & 0.2353 & 0.4105 \\
\hline Foodbought & 0.0000 & 0.0484 & 0.0829 & 0.0937 & 0.1250 & 0.3773 \\
\hline Catering & 0.0000 & 0.0256 & 0.0525 & 0.0652 & 0.0907 & 0.3337 \\
\hline Tobacco & 0.0000 & 0.0000 & 0.0000 & 0.0213 & 0.0309 & 0.3863 \\
\hline BSGroup2 & 0.0829 & 0.2481 & 0.3474 & 0.3549 & 0.4561 & 0.6622 \\
\hline Housing & 0.0000 & 0.1789 & 0.2665 & 0.2758 & 0.3631 & 0.6234 \\
\hline HHgoods & 0.0000 & 0.0045 & 0.0146 & 0.0396 & 0.0436 & 0.5304 \\
\hline HHservices & 0.0000 & 0.0215 & 0.0361 & 0.0478 & 0.0572 & 0.4360 \\
\hline BSGROUP3 & 0.0146 & 0.0665 & 0.1366 & 0.1691 & 0.2375 & 0.5365 \\
\hline Motoring & 0.0000 & 0.0018 & 0.0871 & 0.1199 & 0.1870 & 0.5229 \\
\hline Fuel & 0.0000 & 0.0251 & 390396 & 0.0492 & 0.0613 & 0.5048 \\
\hline
\end{tabular}




\section{Single Women}

\begin{tabular}{|c|c|c|c|c|c|c|}
\hline Variable & Minimum & 1st Quartile & Median & Mean & 3rd Quartile & maximum \\
\hline number of retired & 0.0000 & 0.0000 & 0.0000 & 0.0000 & 0.0000 & 0.0000 \\
\hline number of earners & 1.0000 & 1.0000 & 1.0000 & 1.0000 & 1.0000 & 1.0000 \\
\hline AgeofHHhead & 18.0000 & 29.0000 & 40.0000 & 41.7008 & 54.0000 & 88.0000 \\
\hline Fridge & 0.0000 & 1.0000 & 1.0000 & 0.9829 & 1.0000 & 1.0000 \\
\hline Washing Machine & 0.0000 & 0.0000 & 1.0000 & 0.7218 & 1.0000 & 1.0000 \\
\hline Centr.Heating & 0.0000 & 0.0000 & 1.0000 & 0.7287 & 1.0000 & 1.0000 \\
\hline $\mathrm{TV}$ & 0.0000 & 1.0000 & 1.0000 & 0.7668 & 1.0000 & 1.0000 \\
\hline Video & 0.0000 & 0.0000 & 0.0000 & 0.4528 & 1.0000 & 1.0000 \\
\hline $\mathrm{PC}$ & 0.0000 & 0.0000 & 0.0000 & 0.0370 & 0.0000 & 1.0000 \\
\hline number of cars & 0.0000 & 0.0000 & 1.0000 & 0.6263 & 1.0000 & 3.0000 \\
\hline number of rooms & 1.0000 & 3.0000 & 4.0000 & 4.2810 & 5.0000 & 11.0000 \\
\hline ln.HHincome & 3.3478 & 4.4325 & 4.9210 & 4.8487 & 5.3068 & 5.8917 \\
\hline BS GROUP1 & 0.0532 & 0.1263 & 0.1747 & 0.1841 & 0.2303 & 0.4023 \\
\hline Food bought & 0.0000 & 0.0736 & 0.1118 & 0.1221 & 0.1612 & 0.3823 \\
\hline Catering & 0.0000 & 0.0133 & 0.0336 & 0.0432 & 0.0637 & 0.2653 \\
\hline Tobacco & 0.0000 & 0.0000 & 0.0000 & 0.0188 & 0.0227 & 0.1990 \\
\hline BS Group2 & 0.1207 & 0.2788 & 0.3695 & 0.3732 & 0.4630 & 0.6626 \\
\hline Housing & 0.0000 & 0.1607 & 0.2571 & 0.2616 & 0.3477 & 0.6275 \\
\hline HH goods & 0.0000 & 0.0156 & 0.0382 & 0.0697 & 0.0913 & 0.6811 \\
\hline HH services & 0.0000 & 0.0291 & 0.0449 & 0.0554 & 0.0672 & 0.4495 \\
\hline BSGROUP3 & 0.0192 & 0.0688 & 0.1293 & 0.1524 & 0.2114 & 0.4798 \\
\hline Motoring & 0.0000 & 0.0000 & 0.0647 & 0.0907 & 0.1467 & 0.4437 \\
\hline Fuel & 0.0000 & 0.0330 & 400505 & 0.0617 & 0.0770 & 0.3572 \\
\hline
\end{tabular}


budget share of food

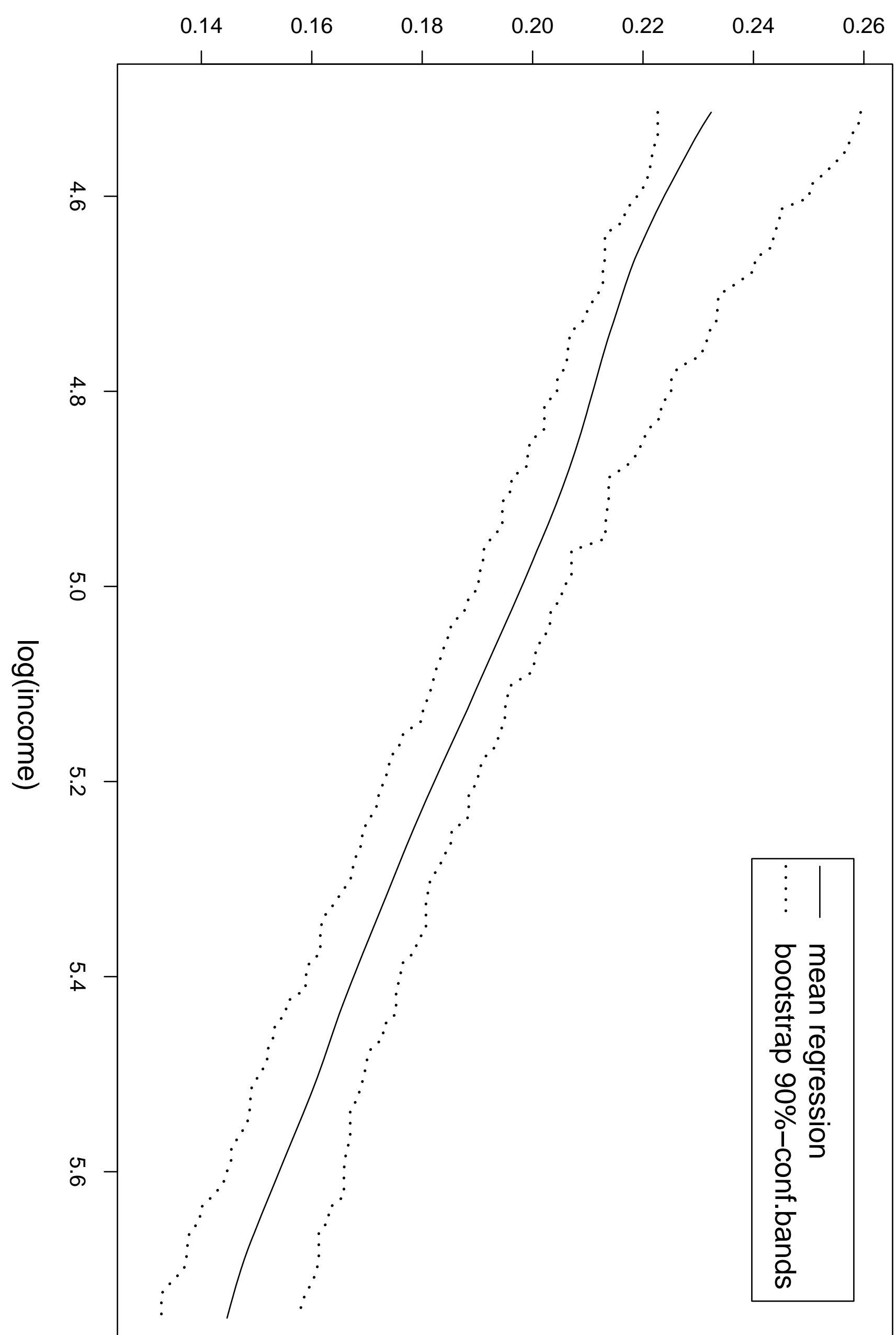




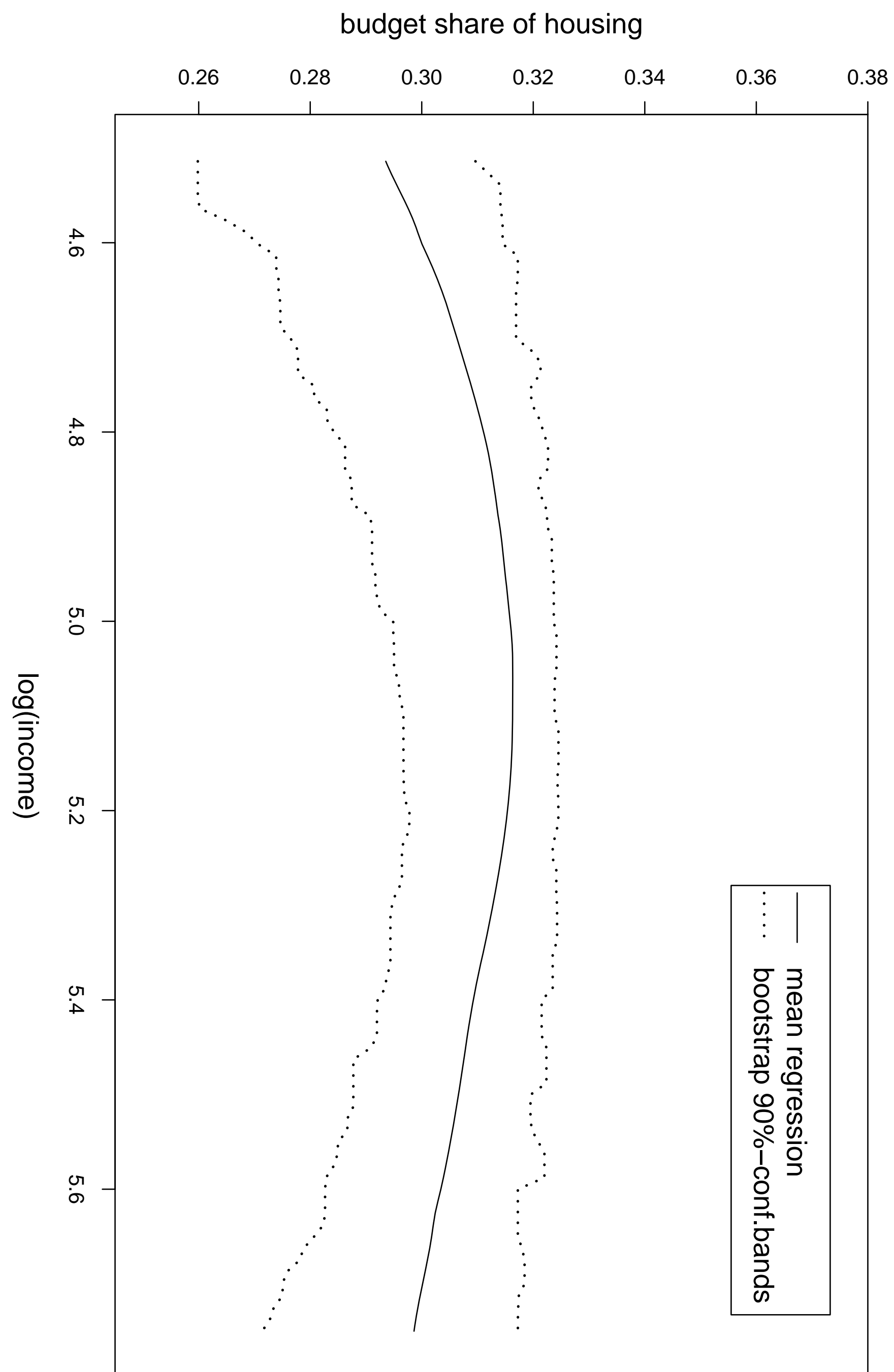


budget share of energy

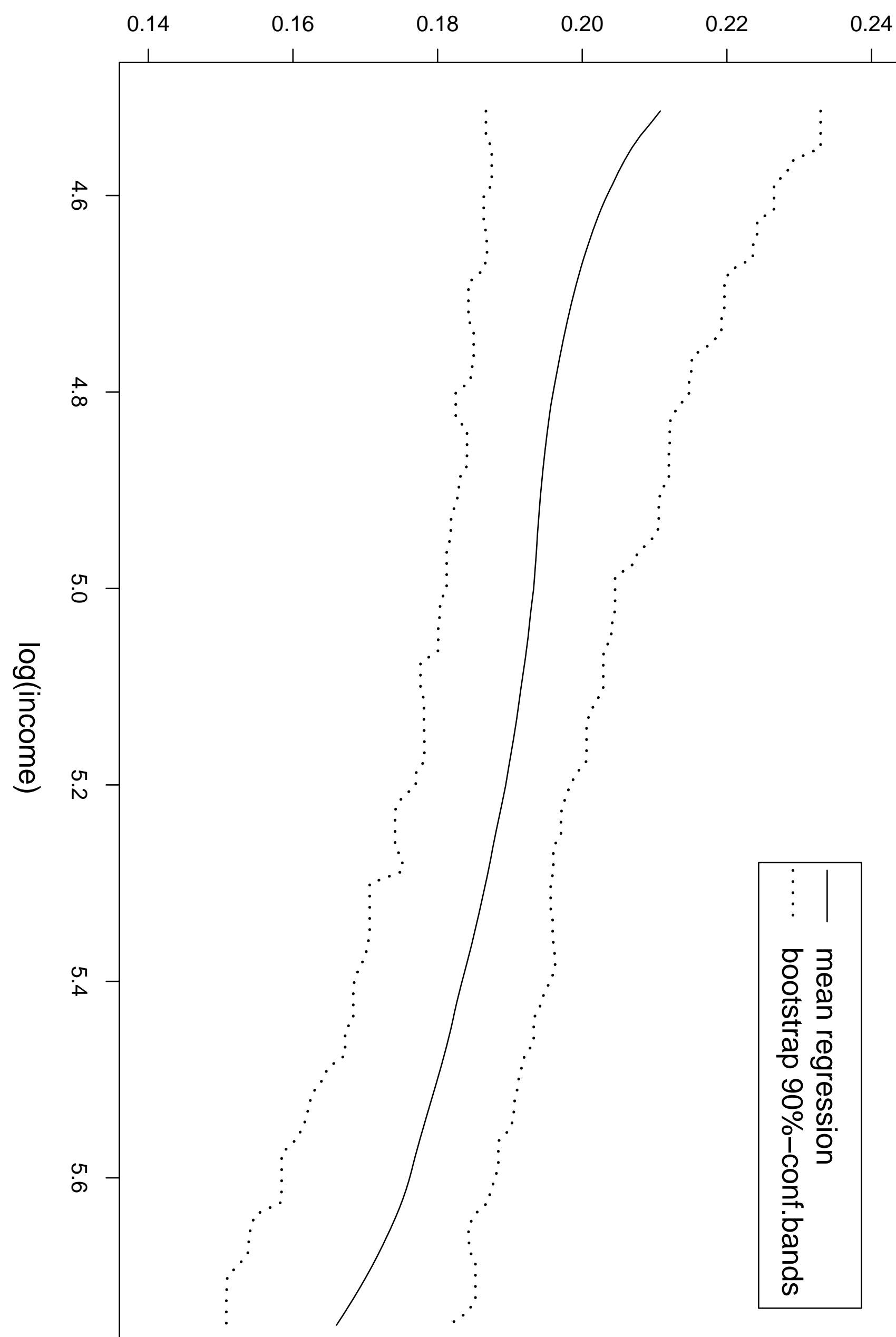


compensated own price effect of food

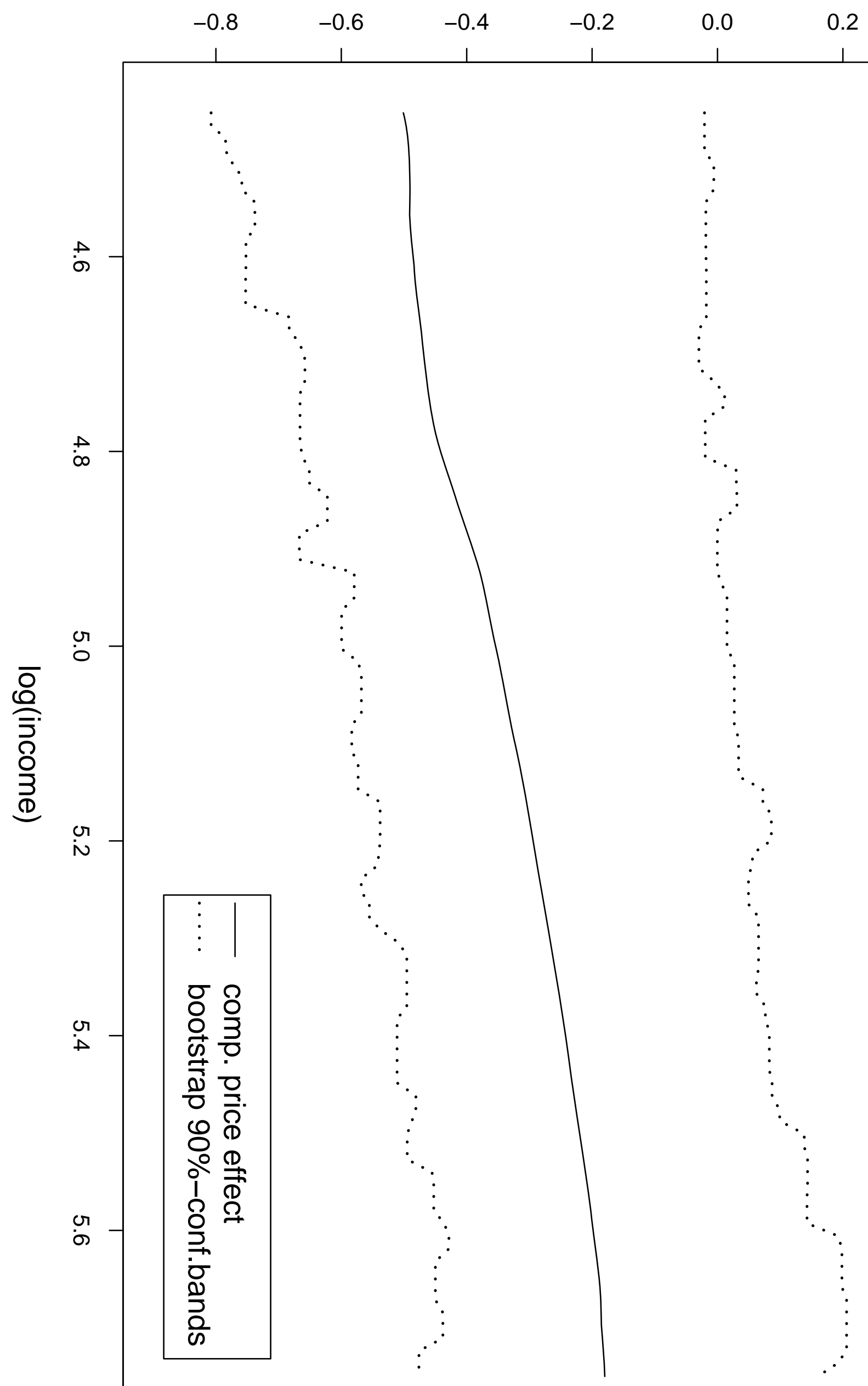


compensated cross price effect of housing price on food demand

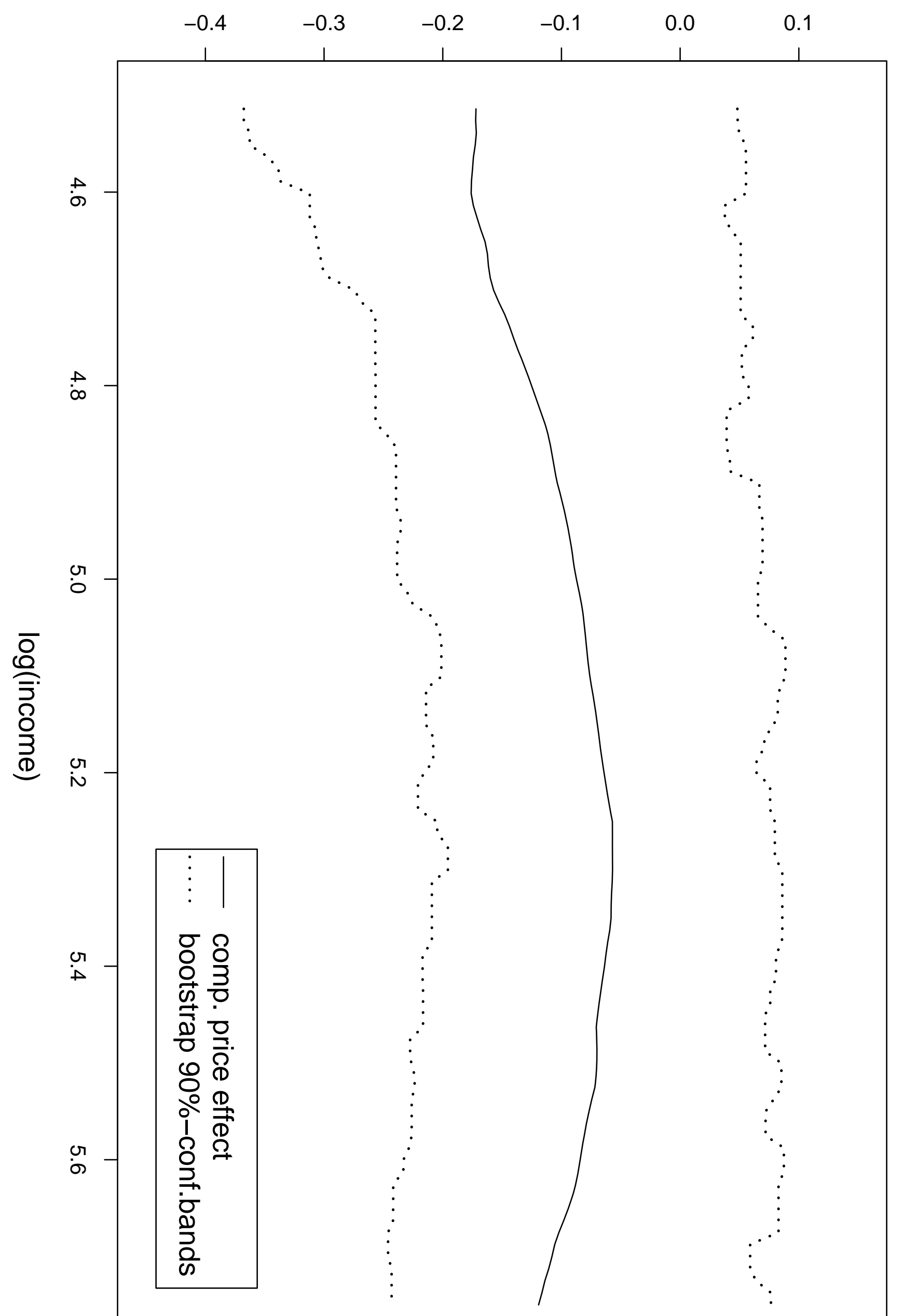


compensated cross price effect of energy price on food demand

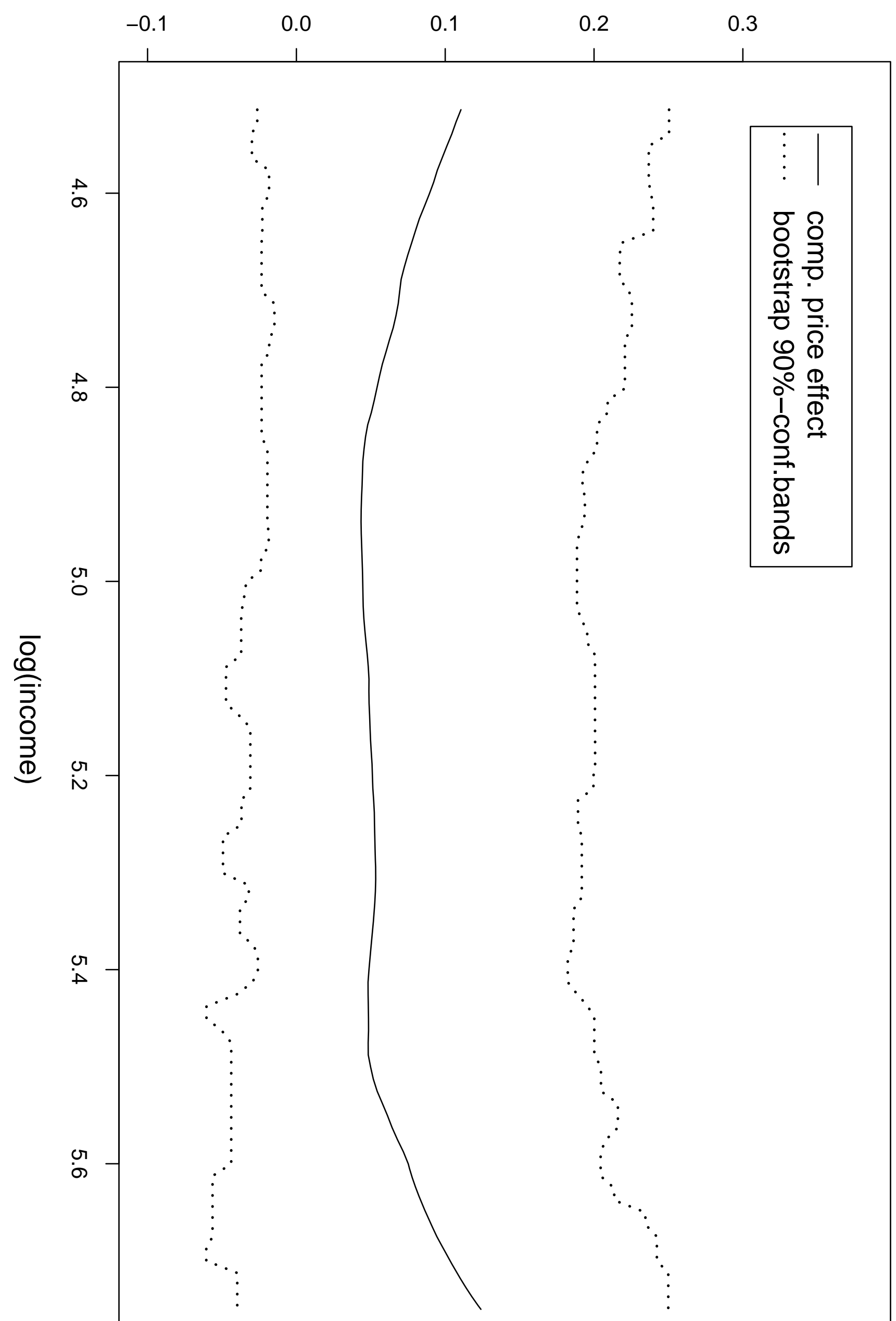


compensated own price effect of housing

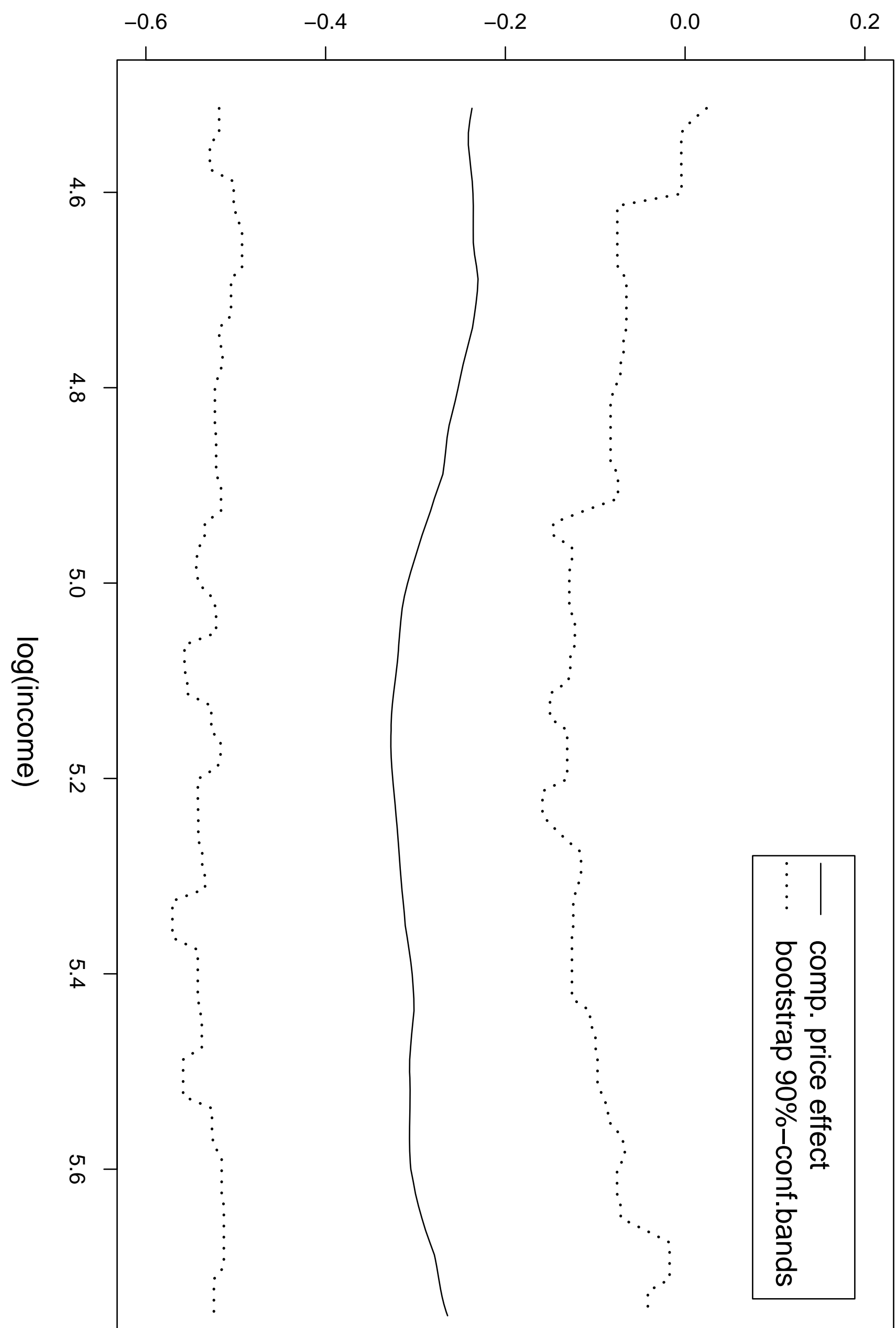


compensated cross price effect of energy price on housing demand

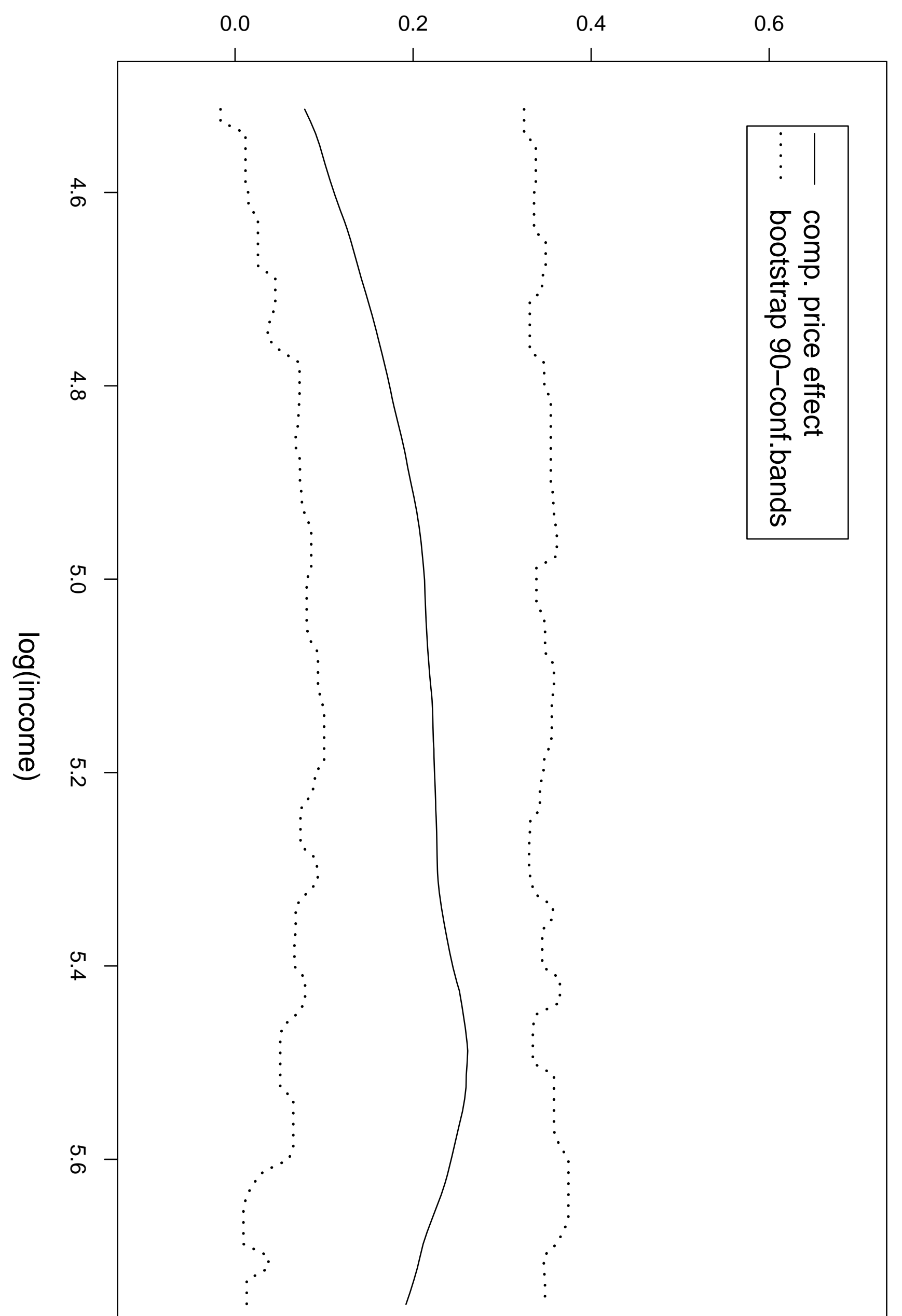


compensated own price effect of energy

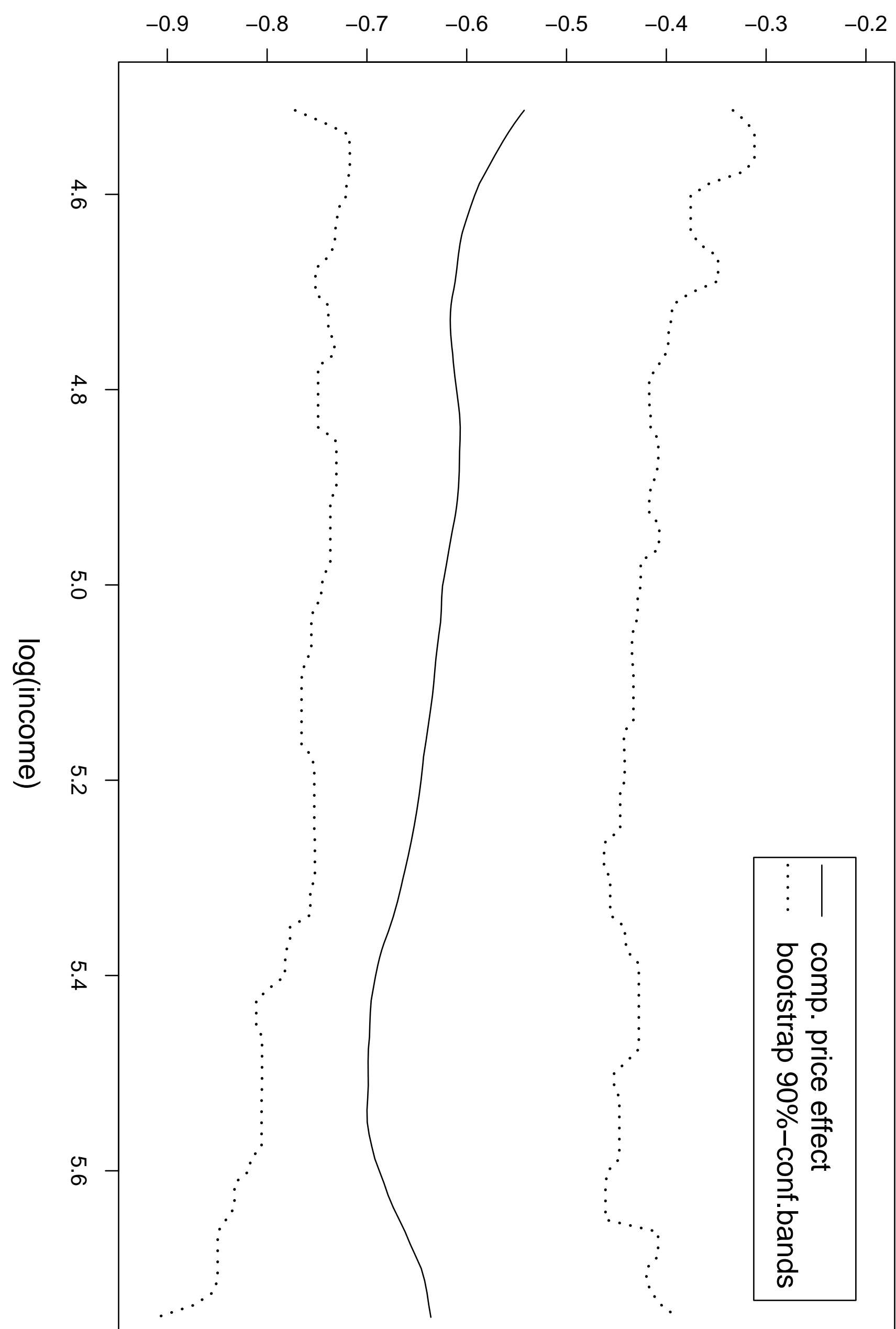




\section{References}

[1] ALtONJI, J., and R. MATZKIN (2005); Cross Section and Panel Data Estimators for Nonseparable Models with Endogenous Regressors, Econometrica, 73, 1053 - 1103.

[2] BLUNDELL, R., BROWNING, M., and I. CRAWFORD (2003); Nonparametric Engel Curves and Revealed Preference, Econometrica, 71, 205-240.

[3] BLUNDELL, R., CHEN, X., and D. KRISTENSEN (2007); Semi-Nonparametric Estimation of Shape Invariant Engel Curves under Endogeneity, Econometrica, forthcoming.

[4] BROWNING, M. and P.-A. CHIAPPORI (1998), Efficient Intra-Household Allocations; A General Characterization and Empirical Tests, Econometrica, 66, 1241 - 1278.

[5] CHERCHYE, L. and F. VERMEULEN (2008), Nonparemtric Analysis of Household Labor Supply; Goodness of Fit and Power of the Unitary and the Collective Model, Review of Economics and Statistics, 90, 267-274.

[6] CHESHER, A. (2003), Identification in Nonseparable Models, Econometrica, 71,1405-1441.

[7] DEATON, A., and J. MUEllBAUER (1980); An almost ideal Demand System, American Economic Review, 70, 312-26.

[8] FORTIN, B. and G. LACROIX (1997); A Test of the Unitary and the Collective Model of Household Labour Supply, Economic Journal, 107, 933-955.

[9] HAAG, B., HODERLEIN, S. and S. MiHAlEVA (2009); Testing Homogeneity of Degree Zero, Working Paper, Brown University.

[10] HAAG, B., HODERLEIN, S. and K. PENDAKUR (2009); Testing and Imposing Slutsky Symmetry, Journal of Econometrics, forthcoming.

[11] HÄRDLE, W., HILDENBRAND, W., and M. JERISON (1991); Empirical Evidence on the Law of Demand, Econometrica, 59, 1525-1549.

[12] HODERLEIN, S. and E. MAMMEN (2007): Identification of Marginal Effects in Nonseparable Models without Monotonicity, Econometrica. 
[13] HODERLEIN, S., KLEMELÄ, J. and E. MAMMEN (2009): Reconsidering the Random Coefficient Model, Econometric Theory, forthcoming.

[14] IMBENS, G. and W. NEWEY (2003), Identification and Estimation of Triangular Simultaneous Equations Models without Additivity, Working Paper, MIT.

[15] JORGENSEN, D., LAU, L. and T. STOKER (1982); The Transcedental Logarithmic Model of Individual Behavior, in: BASMAN, R. and G. RHODES (Eds.), Advances in Econometrics, Vol 1. JAI Press.

[16] KIHLSTROM, R.; A. MAS-COLELL and H. SONNENSCHEIN (1976); The Demand Theory of the Weak Axiom of Revealed Preference, Econometrica, 44, 971-978.

[17] LEWBEL, A. (1999); Consumer Demand Systems and Household Expenditure, in PESARAN, H. and M. WICKENS (Eds.), Handbook of Applied Econometrics, Blackwell Handbooks in economics.

[18] LEWBEL, A. (2001); Demand Systems With and Without Errors, American Economic Review, 611-18.

[19] QUAH, J. (2000), Weak Axiomatic Demand Theory. Working Paper, Nuffield College, Oxford.

[20] STOKER, T. (1989); Tests of Additive Derivative Constraints, Review of Economic Studies, 56, 535-552 